\title{
A Review of Optoelectronic Oscillators for High Speed Signal Processing Applications
}

\author{
Paul Devgan \\ Optical Sciences, NRL, Washington, DC 20032, USA \\ Correspondence should be addressed to Paul Devgan; pdevgan@yahoo.com
}

Received 4 February 2013; Accepted 14 March 2013

Academic Editors: M. Liao, H.-J. Schulze, and J. Solsona

Copyright ( 2013 Paul Devgan. This is an open access article distributed under the Creative Commons Attribution License, which permits unrestricted use, distribution, and reproduction in any medium, provided the original work is properly cited.

\begin{abstract}
The Optoelectronic Oscillator (OEO) was first demonstrated in 1996 as a low phase noise RF source. Low phase noise RF sources have uses for multiple applications, ranging from analog to digital converters to radar to metrology. In the past sixteen years, the OEO has been shown to be useful for other signal processing applications. This paper will provide a background of the OEO's principles of operation, as well as multiple examples of signal processing applications where the OEO can be used. The OEO can be applied to both analog and digital problems, providing new techniques to solve these challenges.
\end{abstract}

\section{Introduction}

As the demand for high speed signal processing increases, methods utilizing photonics have grown in popularity to address this need. High speed signal processing is a general term that incorporates techniques and technologies to address many different applications. Examples of these applications cover a broad range, from analog RF systems such as radars and medical ultrasound imaging, to digital systems covering all the way from long distance communication networks to on-chip interconnects in computers. Photonic signal processing has been shown to address the needs of these systems due to the large instantaneous bandwidth $(>40 \mathrm{GHz})$, low loss $(0.2 \mathrm{~dB} / \mathrm{km}$ in optical fiber $)$ and immunity to electromagnetic interference. One of the common signal processing requirements these varied applications share is the need for very precise timing. Doppler radars require very low phase noise clock sources in order to minimize uncertainty of objects that are being tracked [1]. As optical digital networks have been shown to transmit an aggregate of $10 \mathrm{~Tb} / \mathrm{s}$ of data [2], they require low phase noise clocks for multiplexing the data streams on the entire network. Other applications requiring low phase noise clocks include sampling for analog to digital converters $[3,4]$, clock recovery [5], and pulse sources [6]. The Optoelectronic Oscillator, heretofore referred to as the OEO, is a photonic system that can provide very low phase noise clock signals. In this paper, I will provide some background for the needs for a low phase noise oscillators and present the basic operation of the OEO for low noise signal generation. I will then highlight different demonstrations of the OEO, with an emphasis on different applications to high speed signal processing.

\section{Need for Low Phase Noise Oscillators}

As mentioned above, the need for photonic low phase noise oscillators covers many different applications from very small scales to very large. In computers, on-chip optical interconnects are being investigated to overcome the clock skew and speed limitations that copper interconnects currently exhibit $[7,8]$. In addition, as the processing speeds of the chips increase beyond $1 \mathrm{GHz}$, the requirement on the phase noise of the data clock becomes more stringent. Thus a photonic low phase noise clock that synchronizes the data bus on the chip becomes very important. On the opposite end, optical networks are used to send voice, video, and internet data of $10 \mathrm{~Gb} / \mathrm{s}$ or higher over thousands of kilometers [9]. The ability to synchronize these signals for proper routing and processing is essential to meeting the ever increasing demand for bandwidth. Again the low phase noise photonic clock becomes a key part of the network. A final example of the importance of low phase noise photonic clocks is in their use in analog to digital converters (ADCs). The sample and 
hold circuit of the ADC depends on a very low jitter clock to operate correctly. Any error in the timing of the clock can lead to degraded signal to noise ratio for the ADC, which directly affects the effective number of bits [10]. As a measure of this, one can look at the following relationship between timing jitter and signal to noise ratio can be written as $\operatorname{SNR}(\mathrm{dB})=$ $20 \log _{10}\left(1 / 2 \pi f_{\text {rf }} t_{\text {jitter }}\right)$ and $\operatorname{SNR}(\mathrm{dB})=6.02 N_{\text {bits }}+1.78$ [11], where $f_{\mathrm{rf}}$ is the frequency of an input sinusoidal signal to the ADC, $t_{\text {jitter }}$ is the timing jitter of the clock, and $N_{\text {bits }}$ is the effective number of bits of the ADC. Then the following relationship can be derived $t_{\text {jitter }} \approx\left(1 / f_{\text {samp }} 2^{N_{\text {bits }}+2}\right)$, where $f_{\text {samp }}=2 * f_{\mathrm{rf}}$ is the sampling frequency of the ADC. Plotting this relationship in Figure 1 shows the required timing jitter for a given effective number of bits and sampling rate of the $\mathrm{ADC}$. As the ADCs have increased to sampling rates of $1 \mathrm{GHz}$ and higher, the requirement to get even 10 effective number of bits forces the timing jitter to be on the order of tens of femtoseconds. Thus these devices place the most stringent requirements on the phase noise of the clock and are one of the strongest drivers for low phase noise sources.

\section{Phase Noise and Timing Jitter}

Before introducing the basic OEO, a brief overview of phase noise and its relationship to timing jitter is presented. In characterizing oscillators of any type, the common measurement that is made of the frequency fluctuations is called the phase noise measurement. At least two formal definitions of phase noise have been presented in the literature, which can lead to some confusion [12]. However in the case of the OEO, the commonly accepted definition of the phase noise (denoted as $\mathscr{L}(f)$ ) is the single-side band power spectral density (PSD) of the generated RF signal from the OEO. The PSD is normalized to the power of the RF signal and has units of $\mathrm{dBc} / \mathrm{Hz}$. Often, the phase noise is quoted as a single value measured at a frequency offset from the carrier. To show what the general shape of the phase noise spectrum, a theoretical phase noise plot of an OEO is presented in Figure 2. The OEO is dominated by different sources of noise at different frequency offsets [13]. In the $1 \mathrm{~Hz}$ to $10 \mathrm{~Hz}$ frequency offset range, the noise is dominated by environmental fluctuations, caused by temperature variations and acoustic vibrations and has a slope of $f^{-4}$. In the $10 \mathrm{~Hz}$ to $1 \mathrm{kHz}$ frequency offset range, the noise is dominated by flicker phase noise from the $\mathrm{RF}$ amplification stage necessary for most OEOs to oscillate and has a slope of $f^{-3}$. The next frequency range of $1 \mathrm{kHz}$ to $30 \mathrm{kHz}$ is dominated by white phase noise and has a slope of $f^{-2}$. Finally, at frequency offsets of $30 \mathrm{kHz}$ and higher, the phase noise has a flat noise response that is due to the next non-oscillating side mode of the OEO.

For most applications, especially those involving timing of digital signals, timing jitter is the preferred metric for determining the quality of the clock signal. From the measured phase noise spectrum such as the one in Figure 2, the total noise can be derived. In general this is written as

$$
\sigma_{n}=\sqrt{2 \int_{f_{\min }}^{f_{\max }} \mathscr{L}(f) d f}
$$

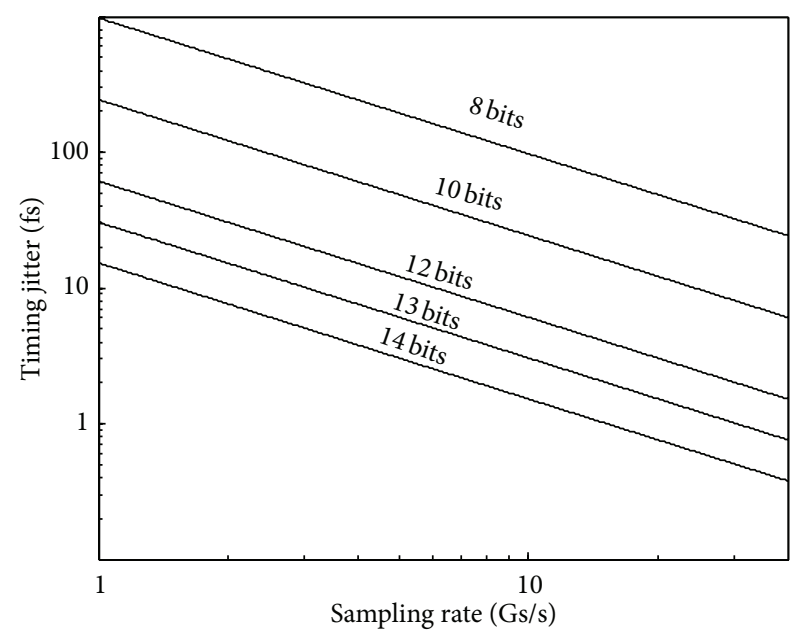

FIGURE 1: Required timing jitter for an analog to digital converter at a sampling rate between 1 and $40 \mathrm{Gs} / \mathrm{s}$ for $8,10,12,13$, and 14 effective number of bits resolution.

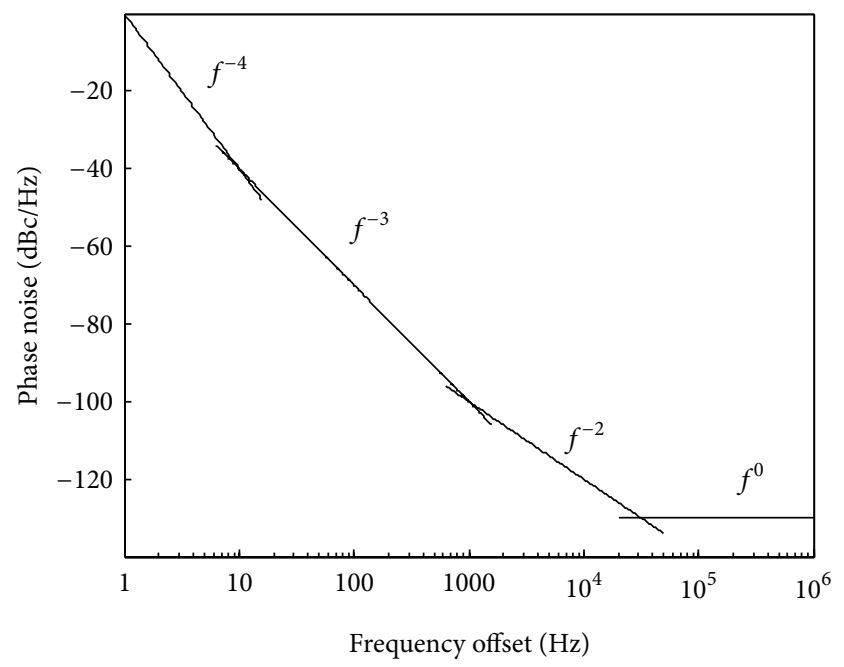

FIgure 2: Theoretical phase noise plot showing the different influences of noise on the power spectral density at frequency offsets from $1 \mathrm{~Hz}$ to $1 \mathrm{Mhz}$ from the carrier.

where $\sigma_{n}$ is the total rms noise and $f_{\max }$ and $f_{\min }$ are the maximum and minimum frequency offsets that the phase noise is integrated over. If the amplitude noise of the signal $\left(\sigma_{a}\right)$ is negligible, the rms timing jitter $\left(\sigma_{j}\right)$ can be obtained by the following relationship $\sigma_{j}=\sigma_{n} /\left(2 \pi f_{\mathrm{rf}}\right)$ with $f_{\mathrm{rf}}$ is the RF frequency of the clock. In the case when the amplitude noise is not negligible, some further work is needed to extract the timing jitter contribution to the total noise. If the higher harmonics of the RF signal can be measured, it has been shown previously [14] that the amplitude noise and timing jitter components can be separated from the overall measured noise by the following relationship $\sigma_{n}^{2}=n^{2}\left(2 \pi f_{\mathrm{rf}}\right)^{2} \sigma_{j}^{2}+\sigma_{a}^{2}$ with $n$ is the $n$-th harmonic of the RF signal being measured. The noise power spectral density of both the timing jitter and the amplitude noise can be seen plotted in Figure 3. Note the 


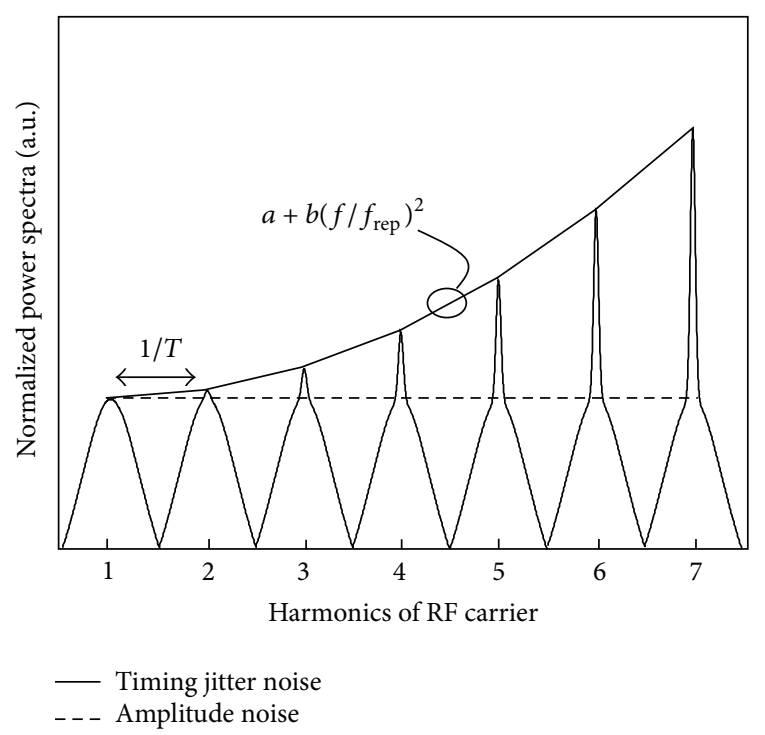

FIGURE 3: Theoretical noise power spectral density for multiple harmonics of the RF frequency showing the timing jitter and amplitude noise contributions.

amplitude noise spectra is arbitrarily plotted broader than the timing jitter noise in order to differentiate the two sources of noise and is not necessarily representative of a real system. As the noise of each harmonic is measured, it will increase quadratically with the timing jitter noise while the amplitude noise contribution is constant. Thus if one can measure the noise of a sufficient number of harmonics and then fit the results to a curve, the timing jitter and amplitude noise contributions can be determined. This is especially applicable to the pulse source OEOs that are described later.

\section{Basic Operation of the OEO}

The use of the OEO for low phase noise signal generation was first proposed by Yao and Maleki [15-17] in 1996. The OEO is similar to the optoelectronic feedback circuits demonstrated by Neyer and Voges [18] in 1982, Nakazawa et al. [19] in 1984, and later by Lewis [20] in 1992. As seen in Figure 4, the OEO starts with a continuous wave laser which is fed into an intensity modulator. The optical output of the intensity modulator is then passed down a long optical fiber delay line and into a photodiode. The recovered electrical signal is then amplified and passed through an electronic bandpass filter. The output of the filter is then connected to the RF input of intensity modulator in order to complete the optoelectronic cavity. When the gain of the cavity is greater than the loss, the OEO will begin to oscillate. The electronic bandpass filter selects the frequency of oscillation by attenuating the other free-running modes of the cavity below threshold.

The OEO differs from the previous optoelectronic circuits by making use of the very low loss of the optical fiber delay line to create a cavity with a very high $Q$ factor. The $Q$ factor can be defined as the ratio of the stored energy in the cavity over the loss of the cavity. Since the loss of the fiber delay line is on the order $0.2 \mathrm{~dB} / \mathrm{km}$, a very long fiber can store a large

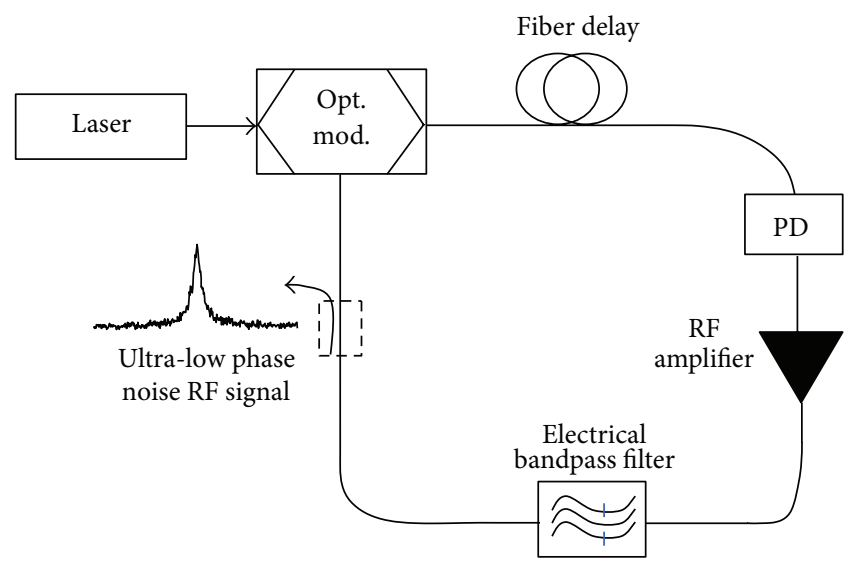

FIgURE 4: Diagram of a basic optoelectronic oscillator. PD: photodiode, Opt. Mod: optical modulator.

amount of energy with very little loss. Because of this the $Q$ factor of the OEO can easily achieve the level of $10^{8}$ or higher. This translates to a $10 \mathrm{GHz}$ clock signal with a phase noise of $-140 \mathrm{dBc} / \mathrm{Hz}$ at $10 \mathrm{kHz}$ offset [15]. Note that there is a limit to the improvement of the phase noise as the length of fiber increases. As shown by Lasri et al. [21], the improvement in the timing jitter, derived from the phase noise as shown in (1), of an OEO has an inverse square root dependence on the fiber length. The limitations are due to the random fiber length variations due to small fluctuations in the environment around the fiber span.

The advantage of the OEO is that the high performance comes with the use of commercially available parts that are inexpensive. Once the first OEO was demonstrated, multiple demonstrations have been presented to improve on the initial design as well as using OEOs for other applications than simply low phase noise RF signal generation.

\section{Multi-Loop OEO}

One of the disadvantages of the initial OEO design was the limitation of the electrical bandpass filter to remove unwanted side modes from the signal. To achieve the highest $Q$ factors for the OEO, the fiber length must be maximized. Unfortunately as the fiber length increases, the spacing between the cavity modes decreases. For example, a $3 \mathrm{~km}$ length of fiber will yield a cavity mode spacing of approximately $67 \mathrm{kHz}$ [21]. A high quality electrical bandpass filter at $10 \mathrm{GHz}$ has a $3 \mathrm{~dB}$ bandwidth of $10 \mathrm{MHz}$ [22]. Thus many non-oscillating side modes will still continue through the electrical bandpass filter and show up in the phase noise measurement. One method to reduce this problem was to introduce a second fiber length into the OEO cavity [23]. An example of this type of OEO is shown in Figure 5. The second loop of the OEO will have its own set of cavity modes. As long as the length of the second loop is not a harmonic multiple of the first loop, the cavity modes will not overlap with each other, as seen below the OEO in Figure 5. However the modes from each loop which are closest to each other will lock and suppress the other cavity modes. This can be seen in Figure 6, 


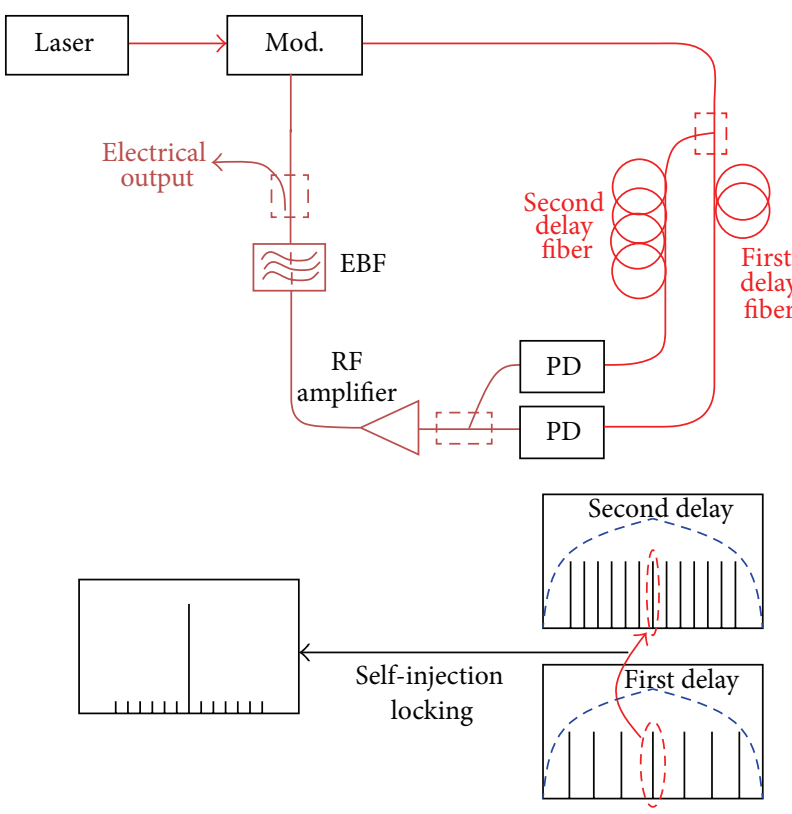

FIGURE 5: Diagram of a dual-loop optoelectronic oscillator along with the cavity modes within the filter passband for the two fiber delays. PD: photodiode, Mod: optical modulator, EBF: bandpass filter.

which shows the single loop phase noise spectrum with the side modes along with the dual loop spectrum with the side modes suppressed below $-110 \mathrm{dBc} / \mathrm{Hz}$. The tradeoff of the system is the phase noise is now an average of noise of the two loops individually, not the phase noise of just the longer loop. In addition, since both loops support the side modes, they are not completely eliminated, but merely suppressed. Nevertheless many demonstrations have been made with the multi-loop OEO in order to suppress the side modes [24-26].

In order to address the shortcomings of the multiloop OEO, a dual cavity OEO was conceived and demonstrated by Zhou and Blasche [27], in which a master-slave configuration was used. In this demonstration, a short loop OEO, known as the slave, is injection locked by a longer loop OEO, known as the master. An example of the master slave OEO appears in Figure 7. Equivalent systems using electronic oscillators have shown that the slave oscillator will have the phase noise performance of the master oscillator. In this case, the overall system has the widely spaced cavity modes of the short loop OEO while having the phase noise performance of the longer loop OEO. The demonstrated phase noise of the generated $10 \mathrm{GHz} \mathrm{RF}$ signal is $-130 \mathrm{dBc} / \mathrm{Hz}$ at an offset of $1 \mathrm{kHz}$ [27]. The non-oscillating side modes are less than $-140 \mathrm{dBc} / \mathrm{Hz}$, which are lower than the side modes in the dual-loop configurations shown previously [23]. This configuration avoids the averaging of the two fiber loops phase noise, with the added complexity of having two entirely separate OEO cavities. Further work has been done to analyze this system more closely and further demonstrations have been made $[28,29]$.

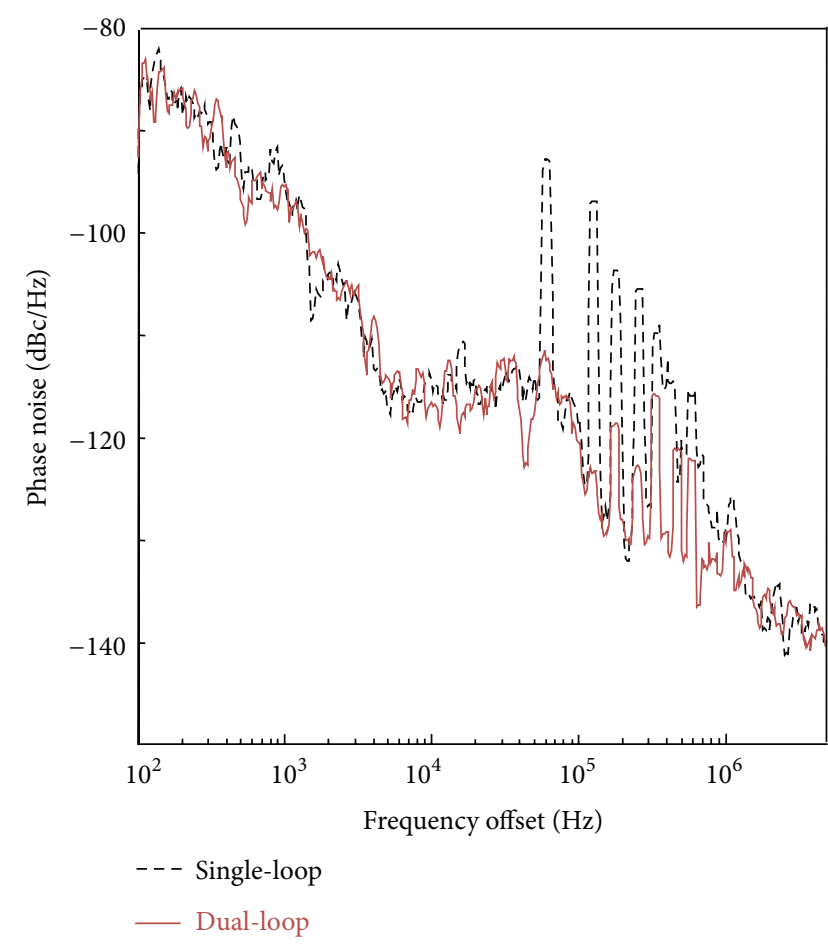

Figure 6: Measured phase noise of the single loop and dual-loop OEO showing the non-oscillating side mode suppression below $-110 \mathrm{dBc} / \mathrm{Hz}$.

\section{OEOs without Electrical Filters}

As previously mentioned, the electrical bandpass filter cannot suppress the non-oscillating sidebands of a long cavity OEO because the modes are too closely spaced together. These side modes are so finely spaced than an electrical filter is unable to suppress them. In addition, the electrical filter is not widely tunable so the RF frequency generated by the OEO is fixed. By removing the filter, the OEO can now be tuned over large frequency ranges. Various methods have been investigated to allow the OEO to oscillate without the use of the electrical bandpass filter. An initial demonstration used the Stimulated Brillouin Scattering (SBS) process to provide a narrow bandwidth gain at $10 \mathrm{GHz}$ to generate an RF signal without the need for the electrical bandpass filter [30]. Further works have proceeded to use this idea to make tunable frequency OEOs since the filter no longer limits the oscillating frequency [31].

Another method is to use an optical filter in the OEO cavity that can have possibly better performance than the electrical bandpass filter. A demonstration using a 1000 finesse Fabry-Perot filter inside the OEO cavity has been shown to select a single frequency [32]. The resulting RF signal has lower phase noise than the same OEO using an electrical bandpass filter. A further demonstration also provides a method for tuning the frequency of the RF signal over a range of $4 \mathrm{MHz}$ by using single side band modulation as opposed to the more common dual side band modulation format [33].

A different demonstration uses the injection locking of a Fabry-Perot laser diode (FP-LD) to generate a certain 


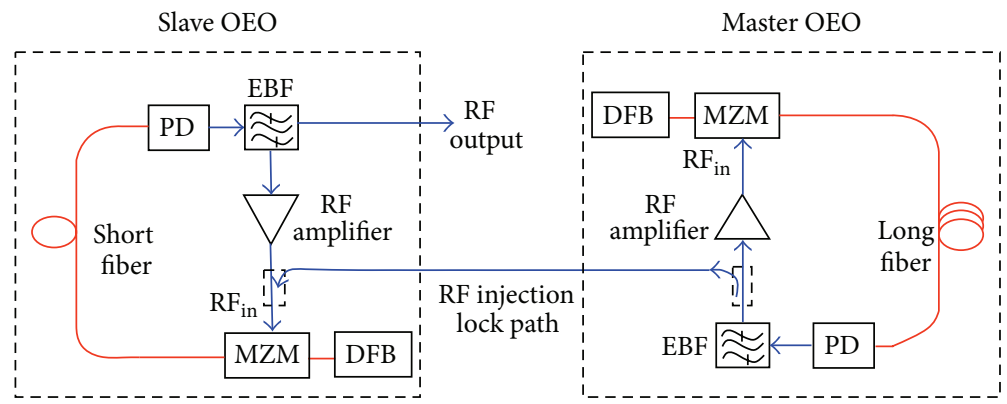

FIGURE 7: Diagram of a master-slave optoelectronic oscillator. DFB: distributed feedback laser, PD: photodiode, MZM: Mach Zehnder modulator, EBF: electrical bandpass filter.

frequency which will then oscillate in the OEO cavity without the need of the electrical bandpass filter. The FP-LD can selectively add gain to one of the modes of the OEO cavity, allowing the chosen one to oscillate while suppressing the others. One of the advantages of this system is that the OEO frequency is now tunable over a $4 \mathrm{GHz}$ range from approximately 6 to $10 \mathrm{GHz}$ [34]. The ability to tune the RF signal frequency comes from changing the wavelength of the FP-LD by tuning its temperature. This in turn changes the oscillation condition of the OEO.

Another configuration for an OEO without an electrical bandpass filter is to use a photonic microwave filter based on recirculating delay lines [35]. By replacing the long single pass fiber delay line in the OEO cavity with two recirculating delay lines, the fiber will act like a microwave filter that supports only a specific oscillating frequency. The oscillating frequency can be changed by changing the length of the two recirculating delay lines. The demonstration shows oscillating frequencies as high as $2.9 \mathrm{GHz}$. To achieve higher frequency oscillations, the use of fiber ring resonators can be used. A demonstration of an OEO with fiber ring resonators and an investigation into the noise properties of these types of OEOs has been made $[36,37]$.

OEOs without an electrical bandpass filter can be useful for other applications. For the OEO to be used in satellites or on computer chips it needs to be miniaturized. Replacing both the long fiber delay and the filter can allow the OEO to be shrunk to chip scale size. One method has used an atomic vapor cell as the frequency selective component in the OEO [38]. For this demonstration, the long fiber delay in the OEO is replaced with a hot rubidium atomic vapor cell. The atomic cell has very narrow electromagnetically induced transparency (EIT) resonances that have been used for atomic reference clocks. These same narrow EIT resonances can be used to stabilize the oscillating frequency of the OEO without the need of an electrical bandpass filter. While an atomic cell OEO does not have the ability to be tuned like the other demonstrations, it can be miniaturized [39].

In another attempt to make a miniaturized OEO while still providing low phase noise RF signal generation, the fiber delay and electrical bandpass filter in the OEO cavity have been replaced with a whispering gallery mode resonator [4042]. Using silicon microspheres, very high-Q cavities can be made in sub-millimeter dimensions that can be integrated with the other components of the OEO in order to put the whole system on a single chip. These types of OEOs have potential applications in satellites for GPS where physical space is at a premium and a precise clock signal is needed to synchronize the GPS signals. Further work has shown that such an OEO can provide low phase noise RF signals without the need for an electrical RF filter [43] at frequencies as high as $34.6 \mathrm{GHz}$.

\section{Optical Pulse Generation OEO}

While the previously described demonstrations of OEOs have focused on generating electrical signals, low phase noise optical pulses have uses for applications in optical networks [2] and in optical sampling [44]. Previous systems have been demonstrated using actively mode locked lasers with very low phase noise. However these demonstrations have utilized very low phase noise RF clocks like the Poseidon oscillator $[45,46]$. Unfortunately the Poseidon oscillator has a fixed frequency output around $10 \mathrm{GHz}$ and is extremely expensive. However the OEO can be used as a cheaper and more frequency flexible solution providing similar phase noise performance. One example of an optical pulse train generated by an OEO is the coupled OEO (C-OEO) presented by Yao et al. [47]. As seen in Figure 8, in this configuration, the intensity modulator in the $\mathrm{OEO}$ is also the same as the one used in an actively-modelocked fiber laser configuration. The pulse repetition rate is determined by the frequency of the OEO and the timing jitter of the optical pulses matches closely to the low phase noise of the RF signal generated by the OEO. A demonstration of the C-OEO showed the phase noise of the optical pulses is $-140 \mathrm{dBc} / \mathrm{Hz}$ at $10 \mathrm{kHz}$ offset [48].

In other configurations, the intensity modulator used in the $\mathrm{OEO}$ is an electro-absorption modulator (EAM) [21]. Due to the nonlinear response of the EAM, optical pulses are generated in the OEO cavity. The advantage of this system as compared to the C-OEO is that the optical pulses and the electrical signal are generated directly from the same cavity, without requiring a separate cavity for the optical pulses. The measured phase noise of the optical pulses matches the phase noise of the electrical RF signal from the OEO. However the width of the pulses generated by the EAM-OEO is limited by the EAM's response. The EAM-OEO has also been 


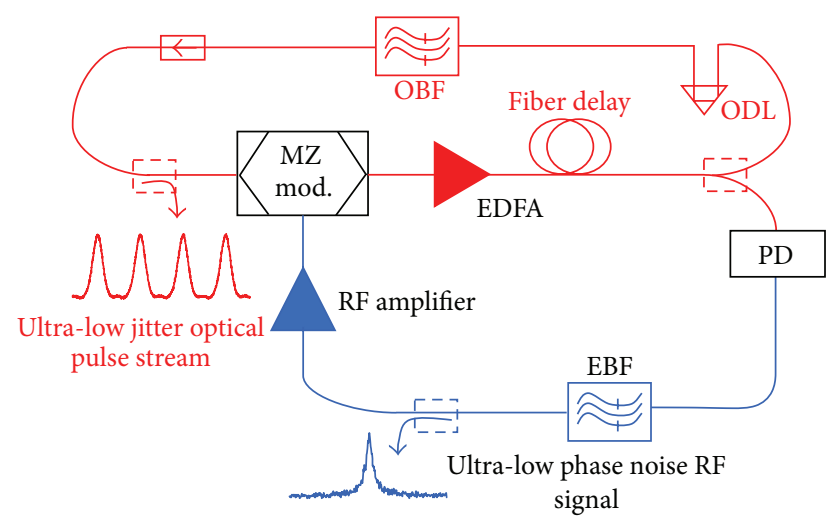

FIGURE 8: Diagram of a coupled optoelectronic for generating optical pulses along with an RF signal. EDFA: erbium-doped fiber amplifier, PD: photodiode, Mod: optical modulator, EBF: bandpass filter, OBF: optical bandpass filter, ODL: optical delay line.

demonstrated with the multi-loop configuration to suppress the non-oscillating side modes [49].

A further advancement of the EAM-OEO as a low phase noise optical pulse source is the ability to generate multiple optical pulse trains, each at a different wavelength, simultaneously [50]. As seen in Figure 9, eight wavelengths are multiplexed together at the input of the EAM. One of the wavelengths is chosen by an optical filter in order to oscillate in the cavity and generate the low phase noise RF signal. Since this same RF signal is used to modulate the wavelengths in the EAM, all of the pulse streams have the same phase noise and they are all synchronized. Such a system is useful for distributing multiple, synchronized, low phase noise clock signals over an optical network. The EAM-OEO has been demonstrated to modulate wavelengths over a range of $100 \mathrm{~nm}$ with the same pulse shape and approximate width. However this system requires multiple lasers at the given wavelengths in order to generate the multiple optical pulse trains.

As stated above the EAM-OEO is limited in pulse width by the response of the EAM and needs a different laser for each wavelength that it wishes to generate. A method to improve upon both these limitations is to use the EAM-OEO in a regeneratively mode locked fiber optic parametric oscillator (RML-FOPO) [51]. The RML-FOPO has the ability to generate two tunable optical pulse streams with very low phase noise and shorter pulse widths from just one laser. The configuration of the RML-FOPO is shown in Figure 10. The pulses have been compressed by $60 \%$ to $70 \%$ (5.9 ps and $3.9 \mathrm{ps})$ as compared to the original pulse widths (15 ps) from the EAM-OEO while the timing jitter has been improved by $68 \%$ ( $230 \mathrm{fs}$ from $710 \mathrm{fs}$ ). The timing jitter and amplitude noise are both improved by using the RML-FOPO as opposed to the FOPO being driven by a commercial signal source. The wavelength range covered by the RML-FOPO is wider than that of a conventional erbium-doped fiber amplifier (EDFA) laser since the RML-FOPO has gain in wavelengths outside the gain spectrum of a traditional EDFA.

While demonstrations have focused on the generation of short optical pulse trains from OEOs, the OEO should be able to generate digital clock pulses that are square. One way to do this is to directly modulate the laser as opposed to using an external modulator. In the case of direct laser modulation, the use of a vertical cavity surface emitting laser (VCSEL) in an OEO is of particular interest [52]. The VCSEL was chosen as this was a laser that was being looked at for use in short distance optical interconnects for computer architectures [53]. By biasing the laser at different points, different optical pulse streams can be generated. As seen in Figure 11, the VCSEL-OEO can generate both sinusoidal RF signals as well as square clock pulses. In order to determine the timing jitter, the phase noise is measured and the total noise is then calculated using (1). Since the source has multiple harmonics that are measurable, the timing jitter and amplitude noise components are 749 fs and 3\%, respectively. Again the multi-loop configuration can be used to suppress the non-oscillating side modes [49]. The side modes are suppressed by about $15 \mathrm{~dB}$ in this case. Work using $850 \mathrm{~nm}$ VCSELs in OEOs continues to be explored [54].

Other methods exist to generate short optical pulses from an OEO. One example uses a heterojunction bipolar phototransistor (photo-HBT) to drive a mode-locked diode laser [55]. The resulting optical pulses are $15 \mathrm{ps}$ wide with a $10 \mathrm{GHz}$ repetition rate. An advancement of this uses the photo-HBT in a coupled OEO with an intracavity fiber optic parametric optical amplifier (FOPA) as the gain medium [56], as opposed to the EDFA used in the original C-OEO. The FOPA's gain response time is on the order of a few femtoseconds, as opposed to the gain response time of a few milliseconds for the EDFA. Thus the optical pulses at $10 \mathrm{GHz}$ can be compressed to as low as 3 ps wide when the proper conditions are met. Even though the pulses are compressed, the measured phase noise is still less than $-100 \mathrm{dBc} / \mathrm{Hz}$ at a $1 \mathrm{kHz}$ frequency offset. Overall there continues to be a strong interest in using OEOs for optical pulse generation with low phase noise.

\section{OEO with Other Optical Modulators}

While the main focus has been on intensity modulation using either an MZM or an EAM as the optical modulator in the OEO cavity, other types of modulators can be used. A popular choice for OEOs has been the phase modulator. As implied by the name, the phase modulator changes the phase of the optical carrier rather than amplitude. This results in a constant intensity optical output from the phase modulator. One of the advantages of the phase modulator is that it requires no DC bias control as is necessary with an MZM intensity modulator. Since the photodetector will not respond to phase changes in the optical carrier, the phase modulation has to be converted to intensity modulation before photodetection. Using either a filter offset from the carrier wavelength or an asymmetric Mach Zehnder (AMZ) interferometer, the phase modulated signal can be converted to an intensity modulated signal. In one demonstration, the offset filter is used in a phase modulated OEO in order to achieve oscillation [57]. The phase modulator is driven with a very large amplitude RF signal in order to generate multiple 


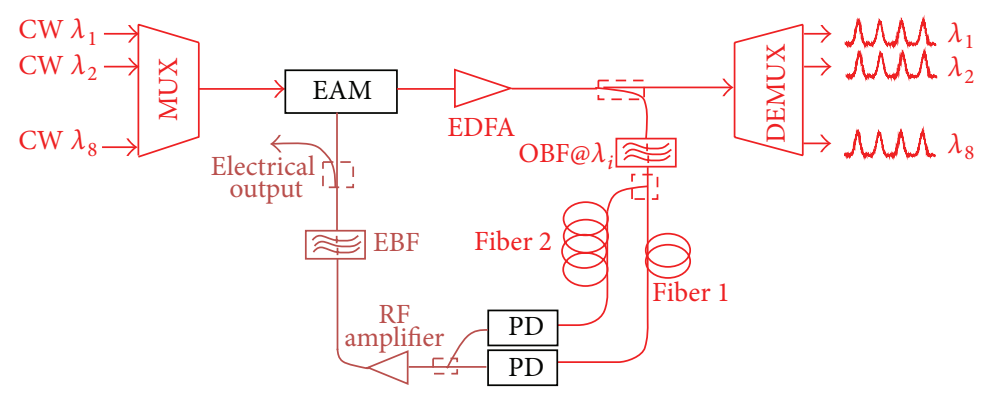

FIGURE 9: Diagram of a multi-wavelength optoelectronic oscillator for generating multiple optical pulse streams with low phase noise. EDFA: erbium-doped fiber amplifier, PD: photodiode, EAM: electro-absorption modulator, EBF: electrical bandpass filter, OBF: optical bandpass filter, MUX: optical wavelength multiplexer, DEMUX: optical wavelength demultiplexer.

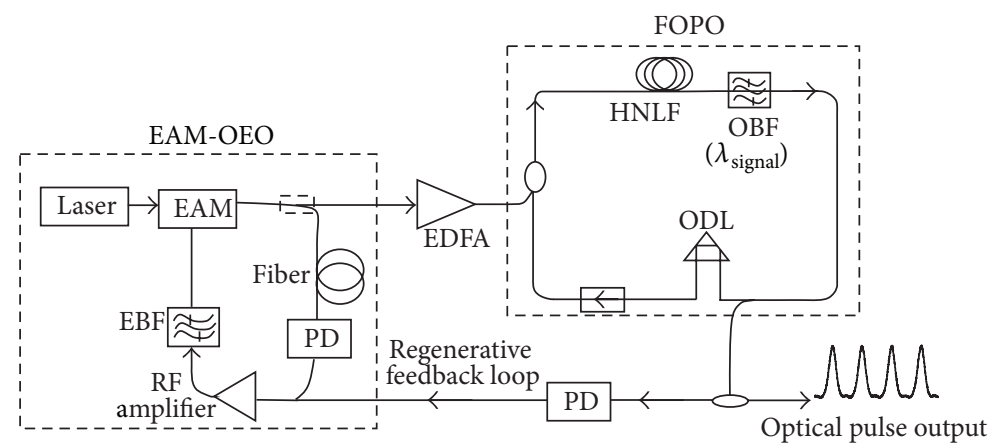

FIGURE 10: Diagram of a regeneratively modelocked fiber optic parametric oscillator using an EAM optoelectronic oscillator for generating short optical pulses with low phase noise. EDFA: erbium-doped fiber amplifier, PD: photodiode, EAM: electro-absorption modulator, EBF: electrical bandpass filter, OBF: optical bandpass filter, MUX: optical wavelength multiplexer, DEMUX: optical wavelength demultiplexer.

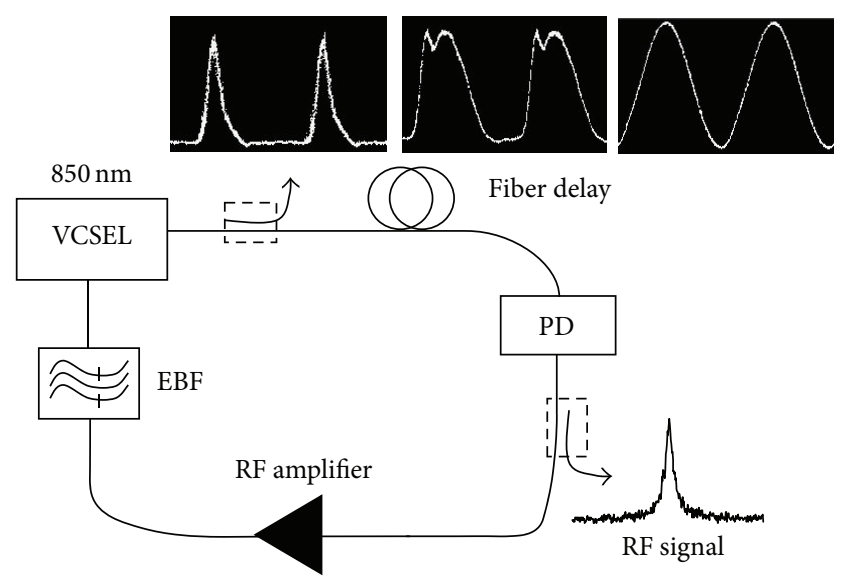

FIGURE 11: Diagram of a VCSEL optoelectronic oscillator for generating different optical pulses with low phase noise. VCSEL: vertical cavity surface emitting laser, PD: photodiode, EBF: electrical bandpass filter.

harmonics. The generated frequency comb has a spacing of $9.95 \mathrm{GHz}$ and a $10 \mathrm{~dB}$ bandwidth of $120 \mathrm{GHz}$. Other methods have used phase modulators to make very stable OEOs [5861].

A different modulator for use in an OEO is the polarization modulator. In this case the polarization modulator changes the polarization state of the optical carrier. By using a polarization sensitive device, such as a polarization beam splitter, the polarization modulation can be converted to intensity modulation. Some of the advantages of using the polarization modulator is that it can be very high speed $(>40 \mathrm{GHz})$ and has lower insertion loss than a traditional MZM. A few demonstrations have been made with this type of modulator in an OEO. The first is a frequency doubling OEO that can generate either a 10 or $20 \mathrm{GHz}$ RF signal from a single OEO cavity without the need for another laser [62]. Another demonstration shows a tunable OEO which can generate an RF signal from 5.8 to $11.8 \mathrm{GHz}$ in $100 \mathrm{MHz}$ steps [63].

While most OEO demonstrations use a laser combined with a modulator, the laser can be directly modulated inside the OEO cavity. In order to increase the oscillating frequency and improve performance, an optically injected OEO (OIL$\mathrm{OEO}$ ) has been demonstrated [64]. In this configuration, a slave laser in the OEO cavity is optically injection locked by a master laser. The output of the slave laser is then detected by a photodiode and the resulting RF signal is then filtered and fed back to the RF input of the slave laser. This allows the OEO to generate a $20 \mathrm{GHz} \mathrm{RF}$ signal without the need for any additional modulator. The resulting phase noise is just above $-110 \mathrm{dBc} / \mathrm{Hz}$ at a $1 \mathrm{~Hz}$ offset. Other OEOs have also been demonstrated using optical injection locking of lasers to generate the RF tone [65]. 


\section{OEO for Clock Recovery and Synchronization}

While OEOs were first used as either low phase noise RF sources or optical pulse generation, the OEO can also be used for other applications, the first one being clock recovery and synchronization. The ability to lock two oscillators so that their outputs are synchronized is achieved by a process known as injection locking. The theory of injection locking is a well-studied subject: after being first discovered by Huygens, who discovered in the late 1800's that the pendulum clocks on the wall of his bedroom would become synchronized if they were placed within a certain distance of each other [66]. The locking mechanism was mechanical vibrations that were transmitted through the wall. Since then injection locking has been observed in many different systems. In fact, the injection locking process is what allows for the multi-loop OEO to combine the RF signal between the different loops to yield a single low noise RF signal. In order to use the OEO for clock recovery, the OEO can be injection locked by an external signal, either RF or an optical data stream. The output of the OEO will then become synchronized with the incoming RF signal. Such a system can also be used with an injected digital pseudo random bit sequence (PRBS) data stream in order to recover the clock of the data. In the case of the OEO, the clock recovery performance can be measured by the resulting phase noise of the injection locked OEO. Before going into more detail of some examples of the demonstrations of clock recovery, I would like to go over some basics of the injection locking process in relation to OEOs.

The injection locking process for electronic oscillators was first formally described by Adler [67]. One oscillator is termed as the injecting oscillator, also called the master oscillator, as it is the source of the signal and the other oscillator is the free-running oscillator, or the slave oscillator, as it is the oscillator to be locked to the injecting oscillator. As long as the frequency of the data stream is within the locking range (a term I will define later) of the slave oscillator, the slave oscillator will become both frequency and phase locked to the incoming data clock. Adler developed mathematical expressions for the locking phenomena between the electronic oscillators and determined the relationship for locking as a function of frequency detuning between the two oscillators. While the initial work involved electronic oscillators, the theory can be expanded to any oscillator, including optical oscillators such as lasers.

As mentioned above, the OEO clock recovery performance can be measured by looking at the phase noise of the OEO when it is injection locked. Following a similar derivation as shown in [68], one can describe the phase noise of the slave OEO in relationship to the phase noise of the injected signal. To begin, the frequency locking range is defined as $\omega_{\text {lock }}=2 \eta \omega_{\mathrm{BW}}$, where $\eta=A_{\text {inj }} / A_{\text {free }}, \omega_{\mathrm{BW}}=\omega_{\text {free }} / 2 Q$, $A_{\text {free }}$ is the free running OEO's signal amplitude, $A_{\text {inj }}$ is the injected signal's amplitude, $Q$ is the quality factor of the OEO, and $\omega_{\text {free }}$ is the free running OEO's frequency. These two components give some insight into the injection locking process of the OEO. First, the higher the $Q$ of the OEO, the narrower the range of frequencies over which the OEO can be injection locked. Secondly, the stronger the injected signal amplitude (or power) is compared to the free running $\mathrm{OEO}$, the wider the locking range is. The locking range can be adjusted by controlling these two parameters for use in a given application. For example, in a SONET optical network, the cutoff frequency for jitter transfer for an OC-192 ( $9.953 \mathrm{~Gb} / \mathrm{s})$ data signal is $120 \mathrm{kHz}$ [69]. So the locking range for an $\mathrm{OEO}$ in this application should be around $120 \mathrm{kHz}$.

Now the phase noise transfer can be discussed. First, $\mathscr{L}_{\text {inj }}(\omega), \mathscr{L}_{\text {free }}(\omega)$, and $\mathscr{L}_{\text {OEO }}(\omega)$ are defined as the phase noise for the injected signal, the free-running slave OEO RF signal, and the injection locked OEO oscillator, respectively. Then looking at the case where the signal is injected into the OEO cavity, the following can be written [49]:

$$
\begin{aligned}
\mathscr{L}_{\mathrm{OEO}}(\omega)= & P_{\text {transfer }}(\omega) \mathscr{L}_{\text {inj }}(\omega) \\
& +P_{\text {transfer }}(\omega) \mathscr{L}_{\text {free }}(\omega)\left(\frac{2 \omega}{\omega_{\text {lock }}}\right)^{2}
\end{aligned}
$$

where $P_{\text {transfer }}(\omega)=1 /\left(1+\left(2 \omega / \omega_{\text {lock }}\right)^{2}\right)$ represents the phase noise transfer function of the oscillator. $P_{\text {transfer }}(\omega)$ has the form of a low-pass filter and thus describes the form of the phase noise when the oscillator is unlocked but is not the same as the free-running noise.

Figure 12 shows the calculated PSD of the phase noise, measured in $\mathrm{dBc} / \mathrm{Hz}$, as a function of offset frequency from the carrier for an injection locked OEO. The frequency-offset range is between $100 \mathrm{~Hz}$ to $10 \mathrm{MHz}$. The phase noise of the free-running OEO and the injected signal both follow an ideal $1 / f^{2}$ relationship (dominated by white noise), with the free-running $\mathrm{OEO}$ phase noise approximately $20 \mathrm{~dB}$ lower than that of the injected signal. For three different locking ranges, the phase noise of the injection locked OEO follows that of the injected signal within the various locking ranges. Outside the locking range, the phase noise decreases to the level of the free running OEO phase noise, following the slope of $P_{\text {transfer }}(\omega)$. With this description in place, some different demonstrations of clock recovery can be presented.

Starting with the EAM-OEO, the same system can be used to recover the clock of a 10 or $40 \mathrm{GHz} \mathrm{RZ}$ digital data signal [70]. The optical signal is injected into the OEO cavity and both an electrical and optical clock are recovered. In order to measure the clock recovery performance, the phase noise of the injected data signal, the free running OEO, and the injected OEO are measured and plotted in Figure 13. Like the theoretical plot in Figure 12, the phase noise of the injected signal and the free running OEO are separate and the phase noise of the injection locked OEO follows the injected signal until about $1 \mathrm{kHz}$ offset and then decreases to the phase noise of the free running OEO. Note that the measured phase noise does decrease with a $-20 \mathrm{~dB} /$ decade slope as predicted by the theory between the $(-85 \mathrm{dBc}$ at $1 \mathrm{kHz}$ offset to $-105 \mathrm{kHz}$ at $10 \mathrm{kHz}$ offset). In order to increase the locking range, the injected optical power into the OEO is increased by approximately $12 \mathrm{~dB}$ while keeping everything else the same. With the higher injected power, the locking range increases just less than 20 times, which again matches the theoretical 


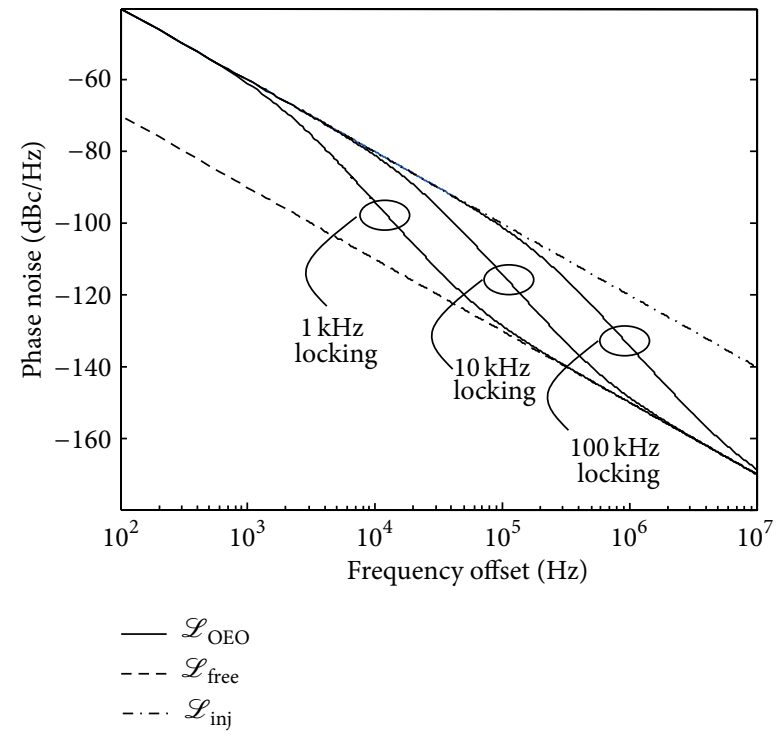

FIGURE 12: Theoretical plot of the phase noise of an injection locked OEO along with the injected signal and free running OEO's phase noise.

prediction. Since the injected OEO phase noise is overall lower than the injected data phase noise, the recovered clock can be used for the retiming portion of an all-optical 3R regenerator. This demonstrates the use of an OEO for clock recovery from an optical data stream.

Another method for injection locking an OEO is to use an electrical signal rather than an optical signal. In this case the VCSEL-OEO is used [49]. A $2 \mathrm{GHz}$ RF signal is electrically injected into the OEO cavity and then time and frequency domain measurements are made to demonstrate that the injected OEO is phase locked to the incoming RF signal. The $\mathrm{RF}$ output of the OEO is split to both the input of an electrical spectrum analyzer (for frequency domain measurements) and the trigger input of a high speed sampling scope (for time domain measurements). A portion of the injected signal is connected to the RF input of the high speed sampling scope. The frequency and time domain plots appear in Figure 14 for three different cases. In the first case, the injected signal's frequency is outside the locking range. The frequency measurement shows the free running signal and the injected signal, along with beat notes between the two. The time measurement shows random noise as the trigger signal is not phase locked to the input signal of the scope. The second case shows the free running OEO and the injected signal almost phase locked. Again the frequency domain measurement shows beat notes and the time domain measurement is still noisy. The third case shows when the OEO and the injected signal are phase locked. Now the frequency measurement shows a single tone and the time domain measurement clearly shows the injected sinusoidal signal. This demonstration shows the OEO generated signal is locked to the injected signal and can be used for synchronization purposes.

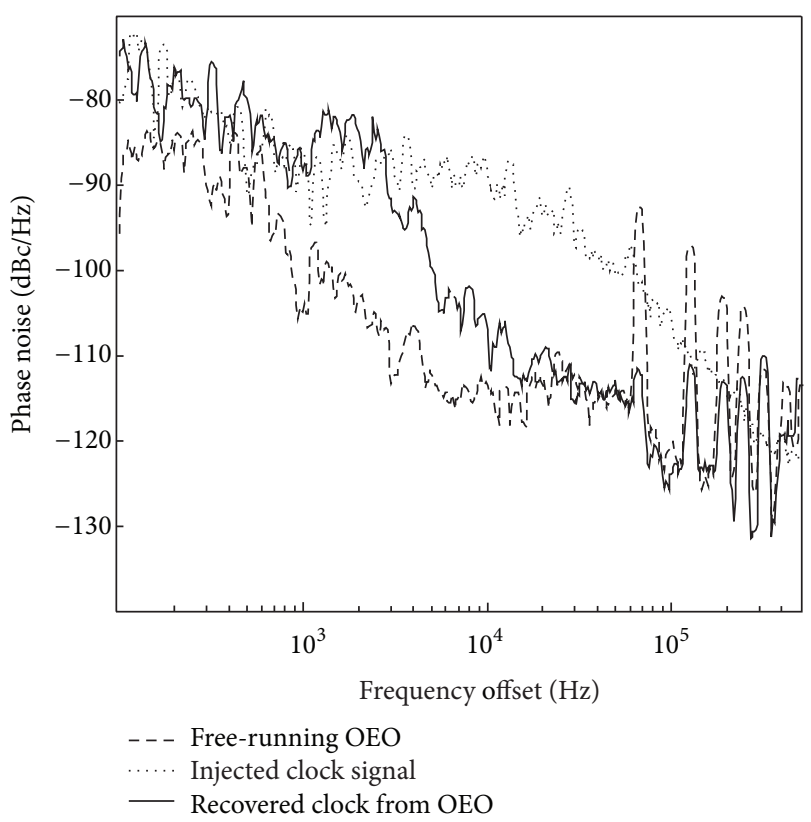

FIGURE 13: Measured plot of the phase noise of an injection locked OEO along with the injected signal and free running OEO's phase noise.

Other examples of injection locked OEOs exist using different modulators, higher frequencies and different injected signals [71-73]. Work continues to use these systems for clock recovery and synchronization for various applications.

\section{OEOs with All-Photonic Gain}

Whether OEOs were used as either low phase noise RF sources or optical pulse generation, one of the limiting noise sources for the OEO is the RF amplifier necessary for the OEO to oscillate. Even though the loss of the optical fiber in the cavity is very low, both the RF to optical conversion at the optical modulator and the optical to RF conversion at the photodetector have loss. Therefore the electronic RF amplifier is necessary to overcome both of these losses. However like any amplifier it will add additional phase noise to the OEO. While a lot of work has been done to reduce the phase noise of the electronic RF amplifiers used in OEOs, they are expensive and not wideband. Therefore each OEO would need its own special RF amplifier to operate at a given frequency, which limits the otherwise potentially wideband operation of an OEO. If the OEO can oscillate with only photonic gain, the wideband operation can be preserved. While other OEOs have been demonstrated without the use of an electronic amplifier [74-76], I will focus on an OEO using an MZM followed by an optical amplifier at $10 \mathrm{GHz}$ using all-photonic gain [77, 78]. The all-photonic RF gain depends on two parameters: the optical power handling of the photodetector and the RF efficiency of the MZM, defined by the half-wave voltage $\left(V_{\pi}\right)$. Both of these parameters are set when the individual device is manufactured and cannot be changed by the user. Without optical or electronic amplification, the highest 

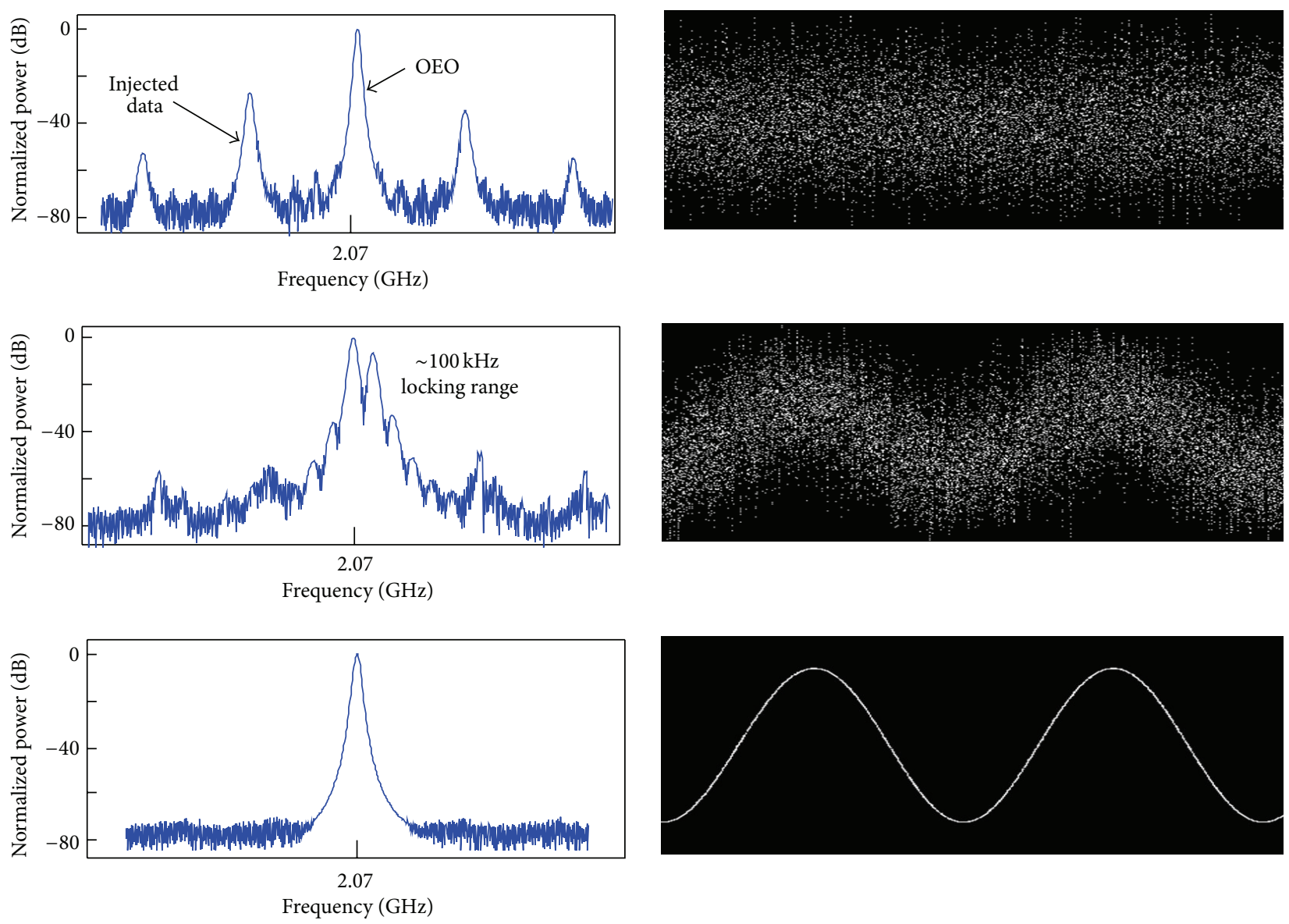

FIGURE 14: Measured plot of the frequency and time domain for the OEO when the injected signal is outside the locking range, just outside the locking range, and when it is injection locked.

all-photonic RF gain for the OEO occurs when the DC bias of the MZM is set to quadrature [77]. In this case the single pass RF gain of the OEO can be defined simply as

$$
G_{\mathrm{RF}}=\left(\frac{I_{\mathrm{dc}}}{V_{\pi}}\right)^{2} \pi^{2} Z_{\mathrm{in}} Z_{\mathrm{out}}
$$

where $I_{\mathrm{dc}}$ is the DC photocurrent from the photodetector, $Z_{\text {in }}$ is the input impedance at the RF port of the MZM and $Z_{\text {out }}$ is the output impedance of the photodetector, both of which are typically $50 \mathrm{ohms}$. Note that $I_{\mathrm{dc}}$ is directly proportional to the input optical power to the photodetector by a factor known as the responsivity of the photodetector. From (3), the RF gain as a function of $I_{\mathrm{dc}}$ can be plotted for an MZM with a $V_{\pi}$ of $2 \mathrm{~V}$ in Figure 15. In this case, the RF gain has to match the loss in the OEO cavity, which is $13 \mathrm{~dB}$. Looking at Figure 15, this would require a photocurrent of approximately $60 \mathrm{~mA}$, which is well above the power handling capabilities of the photodetector. So a different method is required to achieve the required RF gain. By using an optical amplifier after the MZM and then adjusting the DC bias of the MZM, it was shown that the RF gain of the OEO can be increased to a level that matches or exceeds the loss of the OEO cavity. The all photonic gain OEO makes use of the suppression of the optical carrier in order to increase the gain from the optical amplifier. One advantage of the all photonic OEO is the RF power is increased while the photocurrent is kept at $6 \mathrm{~mA}$, a level that the photodetector can handle. Looking at Figure 14, the RF gain at $6 \mathrm{~mA}$ for the all photonic gain is $21.5 \mathrm{~dB}$ higher than in the quadrature bias case. A disadvantage of the all photonic gain OEO is the second harmonic of the RF signal becomes quite large. However in the case of the OEO, the electrical bandpass filter will suppress the second harmonic and make it negligible. The all photonic gain OEO also showed an improvement in the phase noise as compared to the same OEO used with an electrical RF amplifier. The phase noise spectrum shows that the noise is no longer dominated by the flicker phase noise in the $100 \mathrm{~Hz}$ to $1 \mathrm{kHz}$ frequency offset range. In addition the overall phase noise spectrum of the all photonic OEO is lower than in the electronic RF amplifier OEO, in part due to the lower RF noise figure of the overall system. Thus the all photonic gain OEO has several advantages over electronic RF amplifier OEOs.

\section{Millimeter Wave OEOs}

OEOs have continued to leverage the higher bandwidth provided by photonics to generate RF signals in the millimeter wave frequency range. Some examples simply use higher 


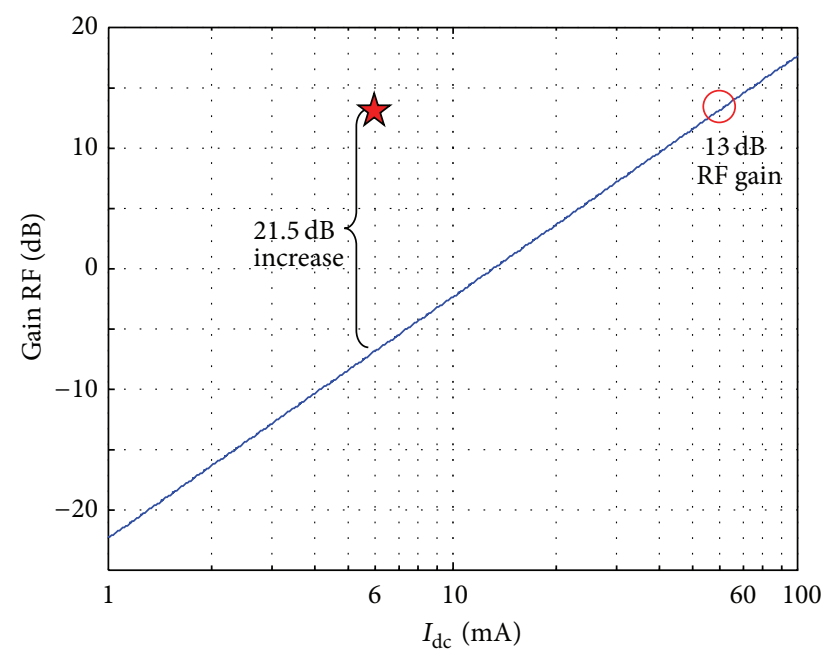

FIGURE 15: Theoretical plot of the RF gain for a single pass in the $\mathrm{OEO}$ as a function of DC photocurrent.

frequency bandwidth devices such as an OEO operating at $39 \mathrm{GHz}$ by using a polymer based modulator [79] and an EAM-OEO that operates at $40 \mathrm{GHz}$ [80]. In this case, the modulator, electrical bandpass filter, photodiode, and electrical RF amplifier all operate in the $40 \mathrm{GHz}$ frequency band. The EAM-OEO can provide a $40 \mathrm{GHz}$ signal with a measured phase noise of $-100 \mathrm{dBc} / \mathrm{Hz}$ at $10 \mathrm{kHz}$ offset, which is $30 \mathrm{~dB}$ better than a commercial RF signal source at the same frequency. The $40 \mathrm{GHz}$ EAM-OEO uses two different lasers with two different wavelengths along with the corresponding zero dispersion fiber in the dual loop cavity. The zero dispersion fiber is necessary as the OEO will not oscillate with standard fiber due to RF fading. The RF fading problem is a well-known problem in long haul photonic links and becomes a problem even at short fiber lengths when the frequency gets to $40 \mathrm{GHz}$. For example, the RF power at $40 \mathrm{GHz}$ for a standard fiber of $1 \mathrm{~km}$ would be about $40 \%$ less than in a zero dispersion fiber. This loss is enough to cause the OEO not to oscillate. By using two different lasers, a single photodetector can be used at the output of the dual loops. As the millimeter wave photodetector is more expensive than a second laser, the OEO can be built for a cheaper cost.

Another way to achieve generation of millimeter wave RF signals is to perform frequency multiplication in the OEO. One example of this expands on the master slave OEO that was described earlier for frequency quadrupling [81]. The key change is that the master OEO operates at $10 \mathrm{GHz}$ and the slave OEO operates at $40 \mathrm{GHz}$. Without the master OEO, the slave OEO has an unstable output that is not usable as a frequency source. On the other hand, when the output of the master OEO is injected into the slave OEO, a stable $40 \mathrm{GHz}$ signal is produced. Other examples of OEOs for millimeter wave generation have gone up to $52.8 \mathrm{GHz}$ [82] by using higher frequency components in the OEO cavity, as well as using mode locked lasers in OEO cavities to generate millimeter wave RF signals [83].

\section{OEOs for Signal Processing of Optical Data Signals}

OEOs have also been shown to be useful for processing of optical data signals. A couple of demonstrations have been shown to convert non-return-to-zero (NRZ) data signals to return-to-zero (RZ) data signals $[84,85]$. In one case, an EAM-OEO is injection locked by the incoming NRZ data signal. By properly adjusting a phase shifter in the cavity, the EAM will carve the NRZ data and create an RZ data stream. Note that since the EAM-OEO has a lower phase noise than the NRZ data stream, the RZ data stream is also re-timed with lower overall timing jitter than the original NRZ signal. The timing jitter is actually improved by up to a factor of 5 times. In addition, the bit error rate (BER) of the RZ signal shows an improvement of $4 \mathrm{~dB}$ in the power response. The system is also polarization independent which is important for real world optical networks where the polarization of the incoming NRZ data streams may have any state of polarization.

Another use for the OEO is to up-convert analog and digital baseband signals onto a higher frequency carrier. In one demonstration [86], a frequency doubled OEO is used to up-convert a $1.25 \mathrm{GHz}$ baseband signal onto the $20 \mathrm{GHz}$ carrier. The up-converted can then be transmitted up to $50 \mathrm{~km}$ without significant penalty. As a further advancement of this system, $161.25 \mathrm{~Gb} / \mathrm{s}$ channels were simultaneously up-converted onto $20 \mathrm{GHz}$ carrier [87]. Each channel was assigned its own wavelength in order to be able to route it to the appropriate wireless base station. Another demonstration uses an $\mathrm{OEO}$ for down-conversion of a $1 \mathrm{~Gb} / \mathrm{s}$ signal from a $20 \mathrm{GHz}$ carrier [88]. The OEO is useful in these applications as it provides higher bandwidth and lower phase noise for the local oscillator signal than a conventional electronic solution.

Yet another use of the OEO is for serial to parallel conversion of optical time domain multiplexed (OTDM) RZ data signals [89]. By use of a polarization modulator along with a CW laser, a single $40 \mathrm{~Gb} / \mathrm{s}$ OTDM data stream can be converted into two $20 \mathrm{~Gb} / \mathrm{s}$ optical data streams along with a $20 \mathrm{GHz}$ prescaled optical clock that is synchronized to the $40 \mathrm{~Gb} / \mathrm{s}$ data stream. The advantage of the polarization modulator is that it allows for serial to parallel conversion with only one OEO. In addition, the polarization modulator provides a constant intensity optical output, as oppose to the intensity modulated output from an EAM or an MZM. Other format conversions are suggested for taking on-off keyed data to quadrature phase shift keyed data. It is clear by these demonstrations that the OEO has been pushed beyond its original purpose and has been used for many different signal processing applications.

\section{OEOs for Selective Amplification of Low Power RF Signals}

While many applications beyond the original intent for the OEO have been provided, the main focus of these demonstrations have been on digital networks, whether for radio over fiber or for format conversion. The OEO does have 
applications in analog systems beyond simply being a low noise RF source. One such application is the detection of low power RF signals in a cluttered environment, which is important in applications ranging from radio astronomy [90] to RF channelization [91]. Imagine a wide frequency spectrum containing multiple RF signals, all with varying powers as shown in Figure 16. In order to pick one of the $\mathrm{RF}$ signals out from the others, it needs to be amplified while the others are suppressed below some chosen threshold level. While an electronic solution could use a very narrow filter combined with an RF amplifier, such a system is hard to realize at microwave frequencies and higher. As already mentioned, electronic filters cannot be made very narrow and RF amplifiers add significant noise. Photonic methods would be preferred as they have very broad instantaneous bandwidths. Specifically, the injection locking process in an OEO can be used to amplify certain RF signals while suppressing the other ones. In this case, the electronic bandpass filter is removed from the OEO cavity, and the power inside the OEO is set just below threshold. Such an OEO will be referred to as a multi-mode OEO (MM-OEO). When an external signal is injected into the OEO cavity, two results can occur. If the injected signal's frequency matches one of the cavity modes of the MM-OEO, it will cause the OEO cavity to oscillate and the signal will see gain. If the injected signal's frequency does not match one of the cavity modes, the signal will see loss and be suppressed. The first demonstration of an MM-OEO used an electronic RF amplifier to show the loss and gain profile over multiple cavity modes [92]. Because the RF amplifier adds noise to the system, the work was extended to an MMOEO that used all photonic gain [93]. Due to the long length of fiber in the EDFA used for the all-photonic gain, the MMOEO cavity modes are spaced about $5.2 \mathrm{MHz}$ apart, with each cavity mode having a gain bandwidth of $1 \mathrm{MHz}$. The MM-OEO has been shown to provide gain for RF signals at frequencies as high as $6 \mathrm{GHz}$. In order to characterize the MM-OEO further, the sensitivity and compression dynamic range are measured. The sensitivity of the MM-OEO is as low as $-83 \mathrm{dBm}$, while the maximum input power into the MM-OEO before the gain becomes compressed is $-11 \mathrm{dBm}$. This yields a compression dynamic range of $72 \mathrm{~dB}$. In order to select a small subset of cavity modes from the MM-OEO, the optical output of the MM-OEO was passed through an integrated silicon Fabry-Perot filter (FPF). The FPF has a $3 \mathrm{~dB}$ bandwidth of $4.5 \mathrm{GHz}$ and after passing the optical signal from the MM-OEO through the FPF, the filtered RF gain is only positive at frequencies of $3 \mathrm{GHz}$ or lower, as opposed to $6 \mathrm{GHz}$ without the FPF. Thus an optical filter can be used to filter the RF response of the MM-OEO. The advantage of the integrated silicon FPF is the ability to fabricate up to 100 filters on a single chip. This scalability in a small size can be advantageous in systems wanting to channelize RF spectrums of multiple gigahertz in bandwidth.

While the MM-OEO described above shows promise for use in selective amplification of RF signals, it is desirable to increase the mode spacing of the MM-OEO cavity, which requires shortening the length of the cavity. As mentioned previously, the EDFA adds an equivalent of approximately 10 meters of fiber into the cavity. In order to overcome this

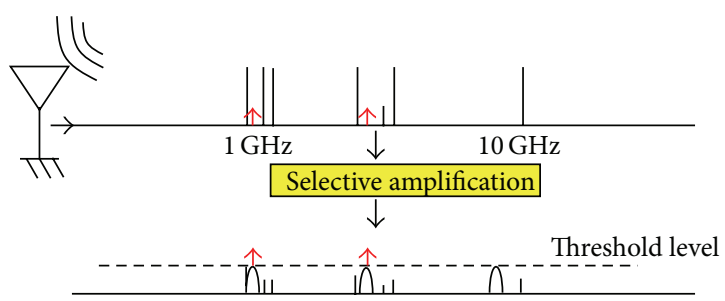

FIGURE 16: RF signals covering 1 to $10 \mathrm{GHz}$ of bandwidth from an antenna before and after selective amplification.

limitation while still using all-photonic gain in the MM-OEO cavity, the EDFA was replaced with a semiconductor optical amplifier (SOA). This device uses a semiconductor gain medium which can be on the order of a centimeter in length, plus the fiber pigtails in order to connect it to the other fiber components in the MM-OEO. Such a length savings can significantly improve the cavity mode spacing. In this new configuration, an MM-OEO was demonstrated having a mode spacing of $100 \mathrm{MHz}$, which is a 20 times improvement over the previous demonstration [94]. While the mode gain bandwidth increases also from $1 \mathrm{MHz}$ to $11 \mathrm{MHz}$, the overall selectivity of this MM-OEO is better than the previous demonstration. Another advantage of increasing the mode spacing allows the MM-OEO to be used with two lasers. By proper selection of the DC bias of the MZM, the two lasers will allow the MM-OEO to switch between complementary modes in the MM-OEO cavity. The modes are complementary in that in one condition, a given frequency will see gain (loss) while in the other condition, the same frequency will now see loss (gain). As seen in Figure 17, with one laser on, one set of modes have gain in the MM-OEO. However when both lasers are on, the complementary set of cavity modes now see gain. This allows the MM-OEO to selectively amplify more frequencies with the same cavity than is possible with just one laser. Another improvement in the system comes from the sensitivity of this MM-OEO. With the increased mode spacing, the integrated noise of the MM-OEO can be measured over $140 \mathrm{MHz}$ while only incorporating one cavity mode. With a measured gain of $10 \mathrm{~dB}$, input sensitivity is calculated to be $-78.4 \mathrm{dBm}$. When compared to thermal noise over the same $14 \mathrm{MHz}$ bandwidth $(-92.6 \mathrm{dBm})$, the MMOEO is only $14.2 \mathrm{~dB}$ above the thermal limit. Thus the MMOEO can be used to amplify very low power RF signals for detection.

The MM-OEO has been shown to offer a method for selectively amplifying low power RF signals. The system can have very good input sensitivity, high compression dynamic range and large selectivity. Further improvements of the MMOEO can be made, the first being to further shorten the cavity in order to get the mode spacing to $1 \mathrm{GHz}$, while increasing the overall bandwidth to $10 \mathrm{GHz}$ and higher. Such improvements can make the MM-OEO attractive to applications such as RF channelization.

\section{Conclusion}

Low phase noise RF oscillators are required in multiple applications, ranging from radar and GPS to high performance 


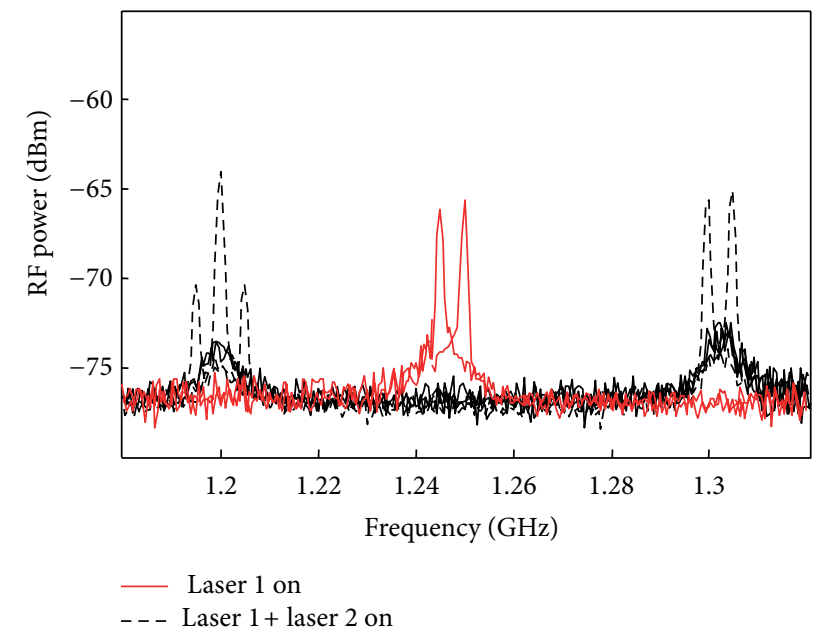

FIgURE 17: The two laser multi-mode optoelectronic oscillator showing selective amplification of complementary modes when either one or both lasers are on from [94].

analog to digital converters to trans-oceanic data transmission. Since 1996, the OEO has been investigated for use in many different applications requiring low phase noise RF signals. Beginning as a low phase noise source, the OEO has been shown to have use in generating low phase noise optical pulse trains and optical clock signals. As the technology has advanced, OEOs have been demonstrated to generate RF signals at frequencies starting in the microwave range and extending out to the millimeter wave range. In addition, work continues on improving the performance of the original OEO, from using multiple loops to suppress the nonoscillating side modes to removing the bandpass filter in order to tune the frequency of the RF output. Further work has been done on improving the phase noise performance by removing the electronic amplifier from the cavity while also looking at miniaturizing the $\mathrm{OEO}$ for use in satellites and computers. Beyond these improvements, the OEO continues to be used for applications beyond its original intent. In terms of digital optical networks, the OEO has been used for both data clock recovery as well as data format conversion. In fact the recovered clock can have lower phase noise then the incoming clock and can then be used to re-time the incoming data signal in order to re-transmit it over further distances. In addition, it has also been shown to be useful for RF signal discrimination. By operating in a multimode configuration just below oscillation threshold, the MM-OEO can be used to selectively amplify low power RF signals in a cluttered environment. The OEO continues to be investigated theoretically [95-97] to better understand the sources of noise as well as to improve their performance. Demonstrations of the OEO continue to explore new applications, from generating broadband chaos [98], use in sensors [99], and as a measurement of the refractive index of optical fibers [100]. Finally OEOs continue to be investigated for use in computer architectures [101]. For the past 16 years the OEO has been shown to have applications in both the RF and digital domain and should continue to provide new insights into future work.

\section{References}

[1] J. R. Vig, "Military applications of high accuracy frequency standards and clocks," IEEE Transactions on Ultrasonics, Ferroelectrics, and Frequency Control, vol. 40, no. 5, pp. 522-527, 1993.

[2] K. Fukuchi, T. Kasamatsu, M. Morie et al., "10.92-Tb/s (273× 40$\mathrm{Gb} / \mathrm{s}$ ) triple-band/ultra-dense WDM optical-repeatered transmission experiment," in Proceedings of the Optical Fiber Communication Conference, p. PD24, March 2001.

[3] B. Shoop, Photonic Analog-to-Digital Conversion, Springer, Berlin, Germany, 2001.

[4] N. Da Dalt, M. Harteneck, C. Sandner, and A. Wiesbauer, "On the jitter requirements of the sampling clock for analog-todigital converters," IEEE Transactions on Circuits and Systems I, vol. 49, no. 9, pp. 1354-1360, 2002.

[5] B. Sartorius, "All-optical clock recovery for 3R optical regeneration," in Proceedings of the Optical Fiber Communication Conference, p. MG7, March 2001.

[6] T. R. Clark, T. F. Carruthers, P. J. Matthews, and I. N. Duling, "Phase noise measurements of ultrastable $10 \mathrm{GHz}$ harmonically modelocked fibre laser," Electronics Letters, vol. 35, no. 9, pp. 720-721, 1999.

[7] A. V. Mule, E. N. Glytsis, T. K. Gaylord, and J. D. Meindl, "Electrical and optical clock distribution networks for gigascale microprocessors," IEEE Transactions on Very Large Scale Integration System, vol. 10, no. 5, pp. 582-594, 2002.

[8] D. A. B. Miller, "Rationale and challenges for optical interconnects to electronic chips," Proceedings of the IEEE, vol. 88, no. 6, pp. 728-749, 2000.

[9] B. Harris and B. Trubey, "10 G-enabled optical network architecture directions for video, voice and data: an MSO perspective," in Proceedings of the Optical Fiber Communication Conference (OFC '06), March 2006.

[10] D. Redmayne, E. Trelewicz, and A. Smith, "Understanding the effect of clock jitter on high speed ADCs," Design Note 1013, Linear Technology, Milpitas, Calif, USA, 2006.

[11] W. Kester, Analog-Digital Conversion, Analog Devices, Norwood, Mass, USA, 2004.

[12] R. Navid, C. Jungemann, T. H. Lee, and R. W. Dutton, "Close-in phase noise in electrical oscillators," in Noise in Communication, vol. 5473 of Proceedings of SPIE, pp. 27-37, Maspalomas, Spain, May 2004.

[13] S. Romisch, J. Kitching, E. Ferre-Pikal, L. Hollberg, and F. L. Walls, "Performance evaluation of an optoelectronic oscillator," IEEE Transactions on Ultrasonics, Ferroelectrics, and Frequency Control, vol. 47, no. 5, pp. 1159-1165, 2000.

[14] D. von der Linde, "Characterization of the noise in continuously operating mode-locked lasers," Applied Physics B, vol. 39, no. 4, pp. 201-217, 1986.

[15] X. S. Yao and L. Maleki, "Optoelectronic oscillator for photonic systems," IEEE Journal of Quantum Electronics, vol. 32, no. 7, pp. 1141-1149, 1996.

[16] X. S. Yao and L. Maleki, "Converting light into spectrally pure microwave oscillation," Optics Letters, vol. 21, no. 7, pp. 483-485, 1996. 
[17] X. S. Yao and L. Maleki, "Optoelectronic microwave oscillator," Journal of the Optical Society of America B, vol. 13, no. 8, pp. 1725-1735, 1996.

[18] A. Neyer and E. Voges, "High-frequency electro-optic oscillator using an integrated interferometer," Applied Physics Letters, vol. 40, no. 1, pp. 6-8, 1982.

[19] M. Nakazawa, T. Nakashima, and M. Tokuda, "An optoelectronic self-oscillatory circuit with an optical fiber delayed feedback and its injection locking technique," Journal of Lightwave Technology, vol. 2, no. 5, pp. 719-730, 1984.

[20] M. F. Lewis, "Novel RF oscillator using optical components," Electronics Letters, vol. 28, no. 1, pp. 31-32, 1992.

[21] J. Lasri, P. Devgan, R. Tang, and P. Kumar, "Self-starting optoelectronic oscillator for generating ultra-low-jitter high-rate (100 GHz or higher) optical pulses," Optics Express, vol. 11, no. 12, pp. 1430-1435, 2003.

[22] K\&L Filter Data Sheet, http://www.klmicrowave.com.

[23] X. S. Yao, L. Maleki, Y. Ji, G. Lutes, and M. Tu, "Dual-loop optoelectronic oscillator," in Proceedings of the IEEE International Frequency Control Symposium (FCS '98), pp. 545-549, May 1998.

[24] X. S. Yao and L. Maleki, "Multiloop optoelectronic oscillator," IEEE Journal of Quantum Electronics, vol. 36, no. 1, pp. 79-84, 2000.

[25] E. Shumakher and G. Eisenstein, "A novel multiloop optoelectronic oscillator," IEEE Photonics Technology Letters, vol. 20, no. 22, pp. 1881-1883, 2008.

[26] T. Bánky, B. Horváth, and T. Berceli, "Optimum configuration of multiloop optoelectronic oscillators," Journal of the Optical Society of America B, vol. 23, no. 7, pp. 1371-1380, 2006.

[27] W. Zhou and G. Blasche, "Injection-locked dual opto-electronic oscillator with ultra-low phase noise and ultra-low spurious level," IEEE Transactions on Microwave Theory and Techniques, vol. 53, no. 3 I, pp. 929-933, 2005.

[28] O. Okusaga, W. Zhou, E. Levy, M. Horowitz, G. Carter, and C. Menyuk, "Experimental and simulation study of dual injectionlocked OEOs," in Proceedings of the IEEE International Frequency Control Symposium (FCS '09), pp. 875-879, April 2009.

[29] O. Okusaga, E. J. Adles, E. C. Levy et al., "Spurious mode reduction in dual injection-locked optoelectronic oscillators," Optics Express, vol. 19, no. 7, pp. 5839-5854, 2011.

[30] X. S. Yao, "High-quality microwave signal generation by use of Brillouin scattering in optical fibers," Optics Letters, vol. 22, no. 17, pp. 1329-1331, 1997.

[31] B. Yang, X. Jin, H. Chi et al., "Optically tunable frequencydoubling Brillouin optoelectronic oscillator with carrier phaseshifted double sideband modulation," IEEE Photonics Technology Letters, vol. 24, pp. 1051-1053, 2012.

[32] I. Ozdur, M. Akbulut, N. Hoghooghi, D. Mandridis, M. U. Piracha, and P. J. Delfyett, "Optoelectronic loop design with 1000 finesse Fabry-Perot etalon," Optics Letters, vol. 35, no. 6, pp. 799-801, 2010.

[33] I. Ozdur, D. Mandridis, N. Hoghooghi, and P. J. Delfyett, "Low noise optically tunable opto-electronic oscillator with fabry perot etalon," Journal of Lightwave Technology, vol. 28, no. 21, pp. 3100-3106, 2010.

[34] S. Pan and J. Yao, "Wideband and frequency-tunable microwave generation using an optoelectronic oscillator incorporating a Fabry-Perot laser diode with external optical injection," Optics Letters, vol. 35, no. 11, pp. 1911-1913, 2010.
[35] X. Liu, W. Pan, X. Zou, B. Luo, L. Yan, and B. Lu, "A reconfigurable optoelectronic oscillator based on cascaded coherencecontrollable recirculating delay lines," Optics Express, vol. 20, pp. 13296-13301, 2012.

[36] K. Saleh, P. H. Merrer, O. Llopis, and G. Cibiel, "Optoelectronic oscillator based on fiber ring resonator: overall system optimization and phase noise reduction," in Proceedings of the IEEE International Frequency Control Symposium (FCS '12), May 2012.

[37] K. Saleh, P. Merrer, O. Llopis, and G. Cibiel, "Optical scattering noise in high $\mathrm{Q}$ fiber ring resonators and its effect on optoelectronic oscillator phase noise," Optics Letters, vol. 37, pp. 518-520, 2012.

[38] D. Strekalov, D. Aveline, N. Yu, R. Thompson, A. B. Matsko, and L. Maleki, "Stabilizing an optoelectronic microwave oscillator with photonic filters," Journal of Lightwave Technology, vol. 21, no. 12, pp. 3052-3061, 2003.

[39] J. Kitching, S. Knappe, and L. Hollberg, "Miniature vapor-cell atomic-frequency references," Applied Physics Letters, vol. 81, no. 3, pp. 553-555, 2002.

[40] V. S. Ilchenko, X. S. Yao, and L. Maleki, "High-Q microsphere cavity for laser stabilization and optoelectronic microwave oscillator," in Laser Resonators II, Proceedings of SPIE, pp. 190198, January 1999.

[41] L. Maleki, V. Iltchenko, S. Huang, and A. Savchenkov, "Micro optical resonators and applications in optoelectronic oscillators," in Proceedings of the IEEE International Topical Meeting on Microwave Photonics (MWP '02), January 2002.

[42] A. B. Matsko, L. Maleki, A. A. Savchenkov, and V. S. Ilchenko, "Whispering gallery mode based optoelectronic microwave oscillator," Journal of Modern Optics, vol. 50, no. 15-17, pp. 25232542, 2003.

[43] A. A. Savchenkov, V. S. Ilchenko, J. Byrd et al., "Whisperinggallery mode based opto-electronic oscillators," in Proceedings of the IEEE International Frequency Control Symposium (FCS '10), pp. 554-557, June 2010.

[44] W. Ng, R. Stephens, D. Persechini, and K. V. Reddy, "Ultra-low jitter modelocking of Er-fibre laser at $10 \mathrm{GHz}$ and its application in photonic sampling for analogue-to-digital conversion," Electronics Letters, vol. 37, no. 2, pp. 113-114, 2001.

[45] L. A. Jiang, M. E. Grein, E. P. Ippen, C. McNeilage, J. Searls, and H. Yokoyama, "Quantum-limited noise performance of a modelocked laser diode," Optics Letters, vol. 27, no. 1, pp. 49-51, 2002.

[46] M. E. Grein, L. A. Jiang, H. A. Haus et al., "Observation of quantum-limited timing jitter in an active, harmonically modelocked fiber laser," Optics Letters, vol. 27, no. 11, pp. 957-959, 2002.

[47] X. S. Yao, L. Davis, and L. Maleki, "Coupled optoelectronic oscillators for generating both RF signal and optical pulses," Journal of Lightwave Technology, vol. 18, no. 1, pp. 73-78, 2000.

[48] N. Yu, E. Salik, and L. Maleki, "Ultralow-noise mode-locked laser with coupled optoelectronic oscillator configuration," Optics Letters, vol. 30, no. 10, pp. 1231-1233, 2005.

[49] P. Devgan, High speed signal processing using nonlinear fibers and optoelectronic devices [PhD dissertation], Northwestern University, Evanston, Ill, USA.

[50] P. Devgan, J. Lasri, R. Tang, and P. Kumar, "Ultra-low-jitter multiwavelength synchronised optical pulse source for C-, Land U-bands," Electronics Letters, vol. 39, no. 18, pp. 1337-1339, 2003.

[51] P. S. Devgan, J. Lasri, R. Tang, V. S. Grigoryan, W. L. Kath, and P. Kumar, "10-GHz dispersion-managed soliton fiber-optical 
parametric oscillator using regenerative mode locking," Optics Letters, vol. 30, no. 5, pp. 528-530, 2005.

[52] P. Devgan, D. Serkland, G. Keeler, K. Geib, and P. Kumar, "An optoelectronic oscillator using an $850 \mathrm{~nm}$ VCSEL for generating low jitter optical pulses," IEEE Photonics Technology Letters, vol. 18, pp. 685-687, 2006.

[53] A. V. Krishnamoorthy, L. M. F. Chirovsky, W. S. Hobson et al., "Vertical-cavity surface-emitting lasers flip-chip bonded to gigabit-per-second CMOS circuits," IEEE Photonics Technology Letters, vol. 11, pp. 128-130, 1999.

[54] H. Hasegawa, Y. Oikawa, and M. Nakazawa, "A 10-GHz optoelectronic oscillator at $850 \mathrm{~nm}$ using a single-mode VCSEL and a photonic crystal fiber," IEEE Photonics Technology Letters, vol. 19, no. 19, pp. 1451-1453, 2007.

[55] J. Lasri, A. Bilenca, D. Dahan et al., "A self-starting hybrid optoelectronic oscillator generating ultra low jitter $10-\mathrm{GHz}$ optical pulses and low phase noise electrical signals," IEEE Photonics Technology Letters, vol. 14, no. 7, pp. 1004-1006, 2002.

[56] D. Dahan, E. Shumakher, and G. Eisenstein, "Self-starting ultralow-jitter pulse source based on coupled optoelectronic oscillators with an intracavity fiber parametric amplifier," Optics Letters, vol. 30, no. 13, pp. 1623-1625, 2005.

[57] T. Sakamoto, T. Kawanishi, and M. Izutsu, "Optoelectronic oscillator using a $\mathrm{LiNbO} 3$ phase modulator for self-oscillating frequency comb generation," Optics Letters, vol. 31, no. 6, pp. 811-813, 2006.

[58] G. J. Dick and Y. Nan, "A new OEO design using optical phase modulation and modulation suppression," in Proceedings of the IEEE International Frequency Control Symposium (FCS '06), pp. 507-512, June 2006.

[59] Y. Jiang, J. Yu, H. Hu, W. Wang, Y. Wang, and E. Yang, "Phasemodulator-based optoelectronic oscillator for generating short optical pulse and microwave signal," Optical Engineering, vol. 46, no. 9, Article ID 090502, 2007.

[60] W. Li and J. Yao, "An optically tunable optoelectronic oscillator," Journal of Lightwave Technology, vol. 28, no. 18, pp. 2640-2645, 2010.

[61] P. T. Callahan, M. L. Dennis, and T. R. Clark, "Experimental demonstration of a phase-modulated optoelectronic oscillator using balanced detection," in Proceedings of the IEEE International Frequency Control and the European Frequency and Time Forum (FCS '11), San Francisco, Calif, USA, May 2011.

[62] S. Pan and J. Yao, "A frequency-doubling optoelectronic oscillator using a polarization modulator," IEEE Photonics Technology Letters, vol. 21, no. 13, pp. 929-931, 2009.

[63] Z. Tang, S. Pan, D. Zhu et al., "Tunable optoelectronic oscillator based on a polarization modulator and a chirped FBG," IEEE Photonics Technology Letters, vol. 24, pp. 1487-1489, 2012.

[64] H. K. Sung, X. Zhao, E. K. Lau, D. Parekh, C. J. Chang-Hasnain, and $\mathrm{M}$. C. Wu, "Optoelectronic oscillators using direct-modulated semiconductor lasers under strong optical injection," IEEE Journal on Selected Topics in Quantum Electronics, vol. 15, no. 3, pp. 572-577, 2009.

[65] M. Haji, L. Hou, A. Kelly et al., "High frequency optoelectronic oscillators based on the optical feedback of semiconductor mode-locked laser diodes," Optics Express, vol. 20, pp. 32683274, 2012.

[66] A. E. Siegman, Lasers, University Science Books, Sausalito, Calif, USA, 1986.

[67] R. Adler, "A study of locking phenomena in oscillators," Proceedings of the IEEE, vol. 61, no. 10, pp. 1380-1385, 1973.
[68] J. Lasri and G. Eisenstein, "Phase dynamics of a timing extraction system based on an optically injection-locked self-oscillating bipolar heterojunction phototransistor," Journal of Lightwave Technology, vol. 20, no. 11, pp. 1924-1932, 2002.

[69] Telcordia Technologies, "Synchronous optical network (SONET) transport systems: common generic criteria," Tech. Rep. GR-253-CORE, Telcordia Technologies, Piscataway, NJ, USA, 2000.

[70] J. Lasri, P. Devgan, R. Tang, and P. Kumar, "Ultralow timing jitter $40-\mathrm{Gb} / \mathrm{s}$ clock recovery using a self-starting optoelectronic oscillator," IEEE Photonics Technology Letters, vol. 16, no. 1, pp. 263-265, 2004.

[71] S. Pan and J. Yao, "Optical clock recovery using a polarizationmodulator-based frequency-doubling optoelectronic oscillator," Journal of Lightwave Technology, vol. 27, no. 16, pp. 35313539, 2009.

[72] H. Tsuchida and M. Suzuki, "40-Gb/s optical clock recovery using an injection-locked optoelectronic oscillator," IEEE Photonics Technology Letters, vol. 17, no. 1, pp. 211-213, 2005.

[73] H. Tsuchida, "Subharmonic optoelectronic oscillator," IEEE Photonics Technology Letters, vol. 20, no. 17, pp. 1509-1511, 2008.

[74] C. W. Nelson, A. Hati, and D. A. Howe, "Microwave optoelectronic oscillator with optical gain," in Proceedings of the IEEE International Frequency Control Symposium (FCS '07), pp. 10141019, May 2007.

[75] W. Zhou, O. Okusaga, C. Nelson, D. Howe, and G. Carter, "10 GHz dual loop opto-electronic oscillator without RF-amplifiers," in Optoelectronic Integrated Circuits X, Proceedings of SPIE, January 2008.

[76] W. Loh, S. Yegnanarayanan, J. Klamkin et al., "Amplifier-free slab-coupled optical waveguide optoelectronic oscillator systems," Optics Express, vol. 20, pp. 19589-19598, 2012.

[77] P. S. Devgan, V. J. Urick, J. D. McKinney, and K. J. Williams, "A low-jitter master-slave optoelectronic oscillator employing all-photonic gain," in Proceedings of the International Topical Meeting on Microwave Photonics (MWP '07), pp. 70-73, October 2007.

[78] P. S. Devgan, V. J. Urick, J. F. Diehl, and K. J. Williams, "Improvement in the phase noise of a $10 \mathrm{GHz}$ optoelectronic oscillator using all-photonic gain," Journal of Lightwave Technology, vol. 27, no. 15, pp. 3189-3193, 2009.

[79] D. H. Chang, H. R. Fetterman, H. Erlig et al., "39-GHz optoelectronic oscillator using broad-band polymer electrooptic modulator," IEEE Photonics Technology Letters, vol. 14, no. 2, pp. 191-193, 2002.

[80] M. Shin, P. S. Devgan, V. S. Grigoryan, P. Kumar, Y. D. Chung, and J. Kim, "Low phase-noise $40 \mathrm{GHz}$ optical pulses from a self-starting electroabsorption-modulator-based optoelectronic oscillator," in Proceedings of the Optical Fiber Communication Conference (OFC '06), Anaheim, Calif, USA, March 2006.

[81] M. Shin and P. Kumar, "Millimeter-wave generation via frequency quadrupling in an optically-injected optoelectronic oscillator," in Proceedings of the Optical Fiber Communication Conference (OFC '07), March 2007.

[82] T. Sakamoto, T. Kawanishi, and M. Izutsu, "Optoelectronic oscillator employing reciprocating optical modulator for millimetre-wave generation," Electronics Letters, vol. 43, no. 19, pp. 1031-1033, 2007.

[83] F. van Dijk, A. Enard, X. Buet, F. Lelarge, and G. H. Duan, "Phase noise reduction of a quantum dash mode-locked laser in 
a millimeter-wave coupled opto-electronic oscillator," Journal of Lightwave Technology, vol. 26, no. 15, pp. 2789-2794, 2008.

[84] L. Huo, Y. Dong, C. Lou, and Y. Gao, "Clock extraction using an optoelectronic oscillator from high-speed NRZ signal and NRZ-to-RZ format transformation," IEEE Photonics Technology Letters, vol. 15, no. 7, pp. 981-983, 2003.

[85] J. Lasri, P. Devgan, V. S. Grigoryan, and P. Kumar, "Multiwavelength NRZ-to-RZ conversion with significant timing-jitter suppression and SNR improvement," Optics Communications, vol. 240, no. 4-6, pp. 293-298, 2004.

[86] M. Shin and P. Kumar, "Optical microwave frequency upconversion via a frequency-doubling optoelectronic oscillator," IEEE Photonics Technology Letters, vol. 19, no. 21, pp. 1726-1728, 2007.

[87] M. Shin and P. Kumar, "Frequency up-conversion of optical microwaves for multichannel optical microwave system on a WDM network," Optical Fiber Technology, vol. 18, pp. 242-246, 2012.

[88] D. Zhu, S. Pan, S. Cai, and D. Ben, "High-performance photonic microwave downconverter based on a frequency-doubling optoelectronic oscillator," IEEE Journal of Lightwave Technology, vol. 30, pp. 3036-3042, 2012.

[89] H. Tsuchida, "Simultaneous prescaled clock recovery and serialto-parallel conversion of data signals using a polarization modulator-based optoelectronic oscillator," Journal of Lightwave Technology, vol. 27, no. 17, pp. 3777-3782, 2009.

[90] J. Raza, A. J. Boonstra, and A. J. van der Veen, "Spatil filtering of RF interference in radio astronomy," IEEE Signal Processing Letters, vol. 9, no. 2, pp. 64-67, 2002.

[91] G. W. Anderson, D. C. Webb, A. E. Spezio, and J. N. Lee, "Advanced channelization for RF, microwave, and millimeterwave applications," Proceedings of the IEEE, vol. 79, no. 3, pp. 355-388, 1991.

[92] V. J. Urick, P. S. Devgan, J. D. McKinney, F. Bucholtz, and K. J. Williams, "Channelisation of radio-frequency signals using optoelectronic oscillator," Electronics Letters, vol. 45, no. 24, pp. 1242-1244, 2009.

[93] P. S. Devgan, M. W. Pruessner, V. J. Urick, and K. J. Williams, "Detecting low-power RF signals using a multimode optoelectronic oscillator and integrated optical filter," IEEE Photonics Technology Letters, vol. 22, no. 3, pp. 152-154, 2010.

[94] P. S. Devgan, V. J. Urick, and K. J. Williams, "Detection of lowpower RF signals using a two laser multimode optoelectronic oscillator," IEEE Photonics Technology Letters, vol. 24, pp. 857859, 2012.

[95] E. C. Levy, M. Horowitz, and C. R. Menyuk, "Modeling optoelectronic oscillators," Journal of the Optical Society of America $B$, vol. 26, no. 1, pp. 148-159, 2009.

[96] Y. K. Chembo, L. Larger, H. Tavernier, R. Bendoula, E. Rubiola, and P. Colet, "Dynamic instabilities of microwaves generated with optoelectronic oscillators," Optics Letters, vol. 32, no. 17, pp. 2571-2573, 2007.

[97] Y. K. Chembo, K. Volyanskiy, L. Larger, E. Rubiola, and P. Colet, "Determination of phase noise spectra in optoelectronic microwave oscillators: a langevin approach," IEEE Journal of Quantum Electronics, vol. 45, no. 2, pp. 178-186, 2009.

[98] K. Callan, L. Illing, Z. Gao, D. Gauthier, and E. Schöll, "Broadband chaos generated by an opto-electronic oscillator," Physical Review Letters, vol. 104, Article ID 113901, 4 pages, 2010.

[99] N. L. Duy, B. Journet, I. Ledoux-Rak, J. Zyss, L. V. H. Nam, and V. van Luc, "Opto-electronic oscillator: applications to sensors," in Proceedings of the IEEE International Topical Meeting on Microwave Photon ((MWP '08), pp. 131-134, October 2008.

[100] L. D. Nguyen, K. Nakatani, and B. Journet, "Refractive index measurement by using an optoelectronic oscillator," IEEE Photonics Technology Letters, vol. 22, no. 12, pp. 857-859, 2010.

[101] C. Gunn, D. Guckenberger, T. Pinguet et al., "A low phase noise $10 \mathrm{GHz}$ optoelectronic RF oscillator implemented using CMOS photonics," in Proceedings of the 54th IEEE International SolidState Circuits Conference (ISSCC '07), pp. 567-622, February 2007. 

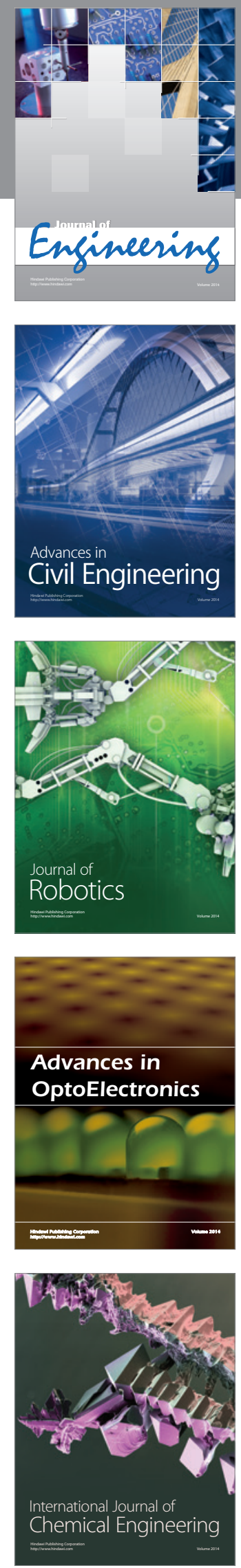

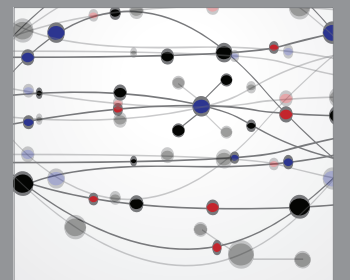

The Scientific World Journal
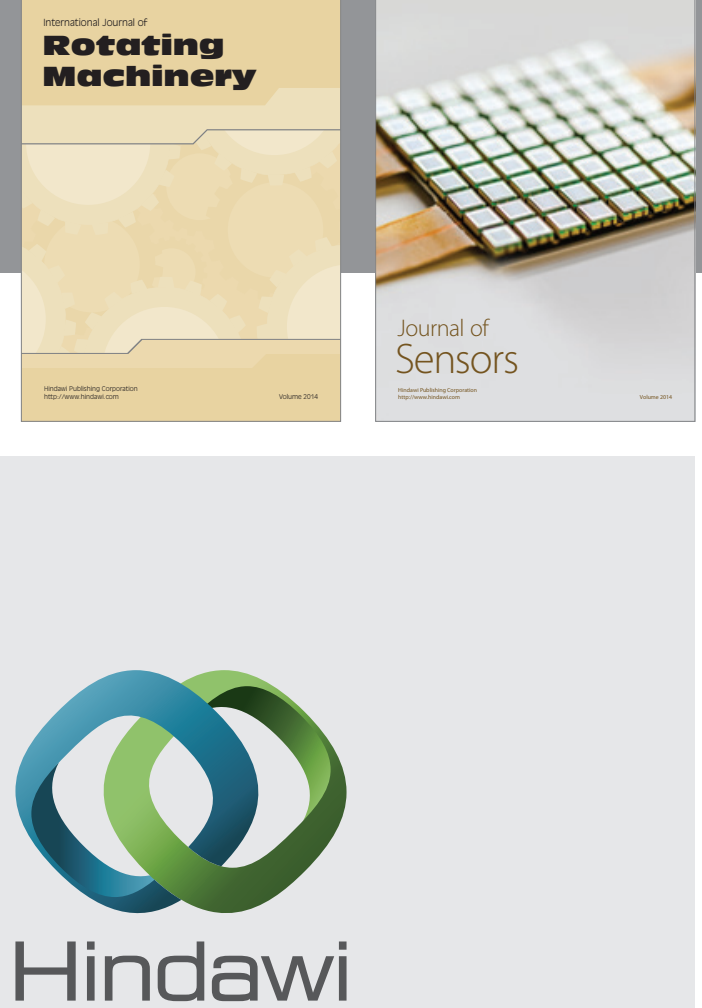

Submit your manuscripts at http://www.hindawi.com
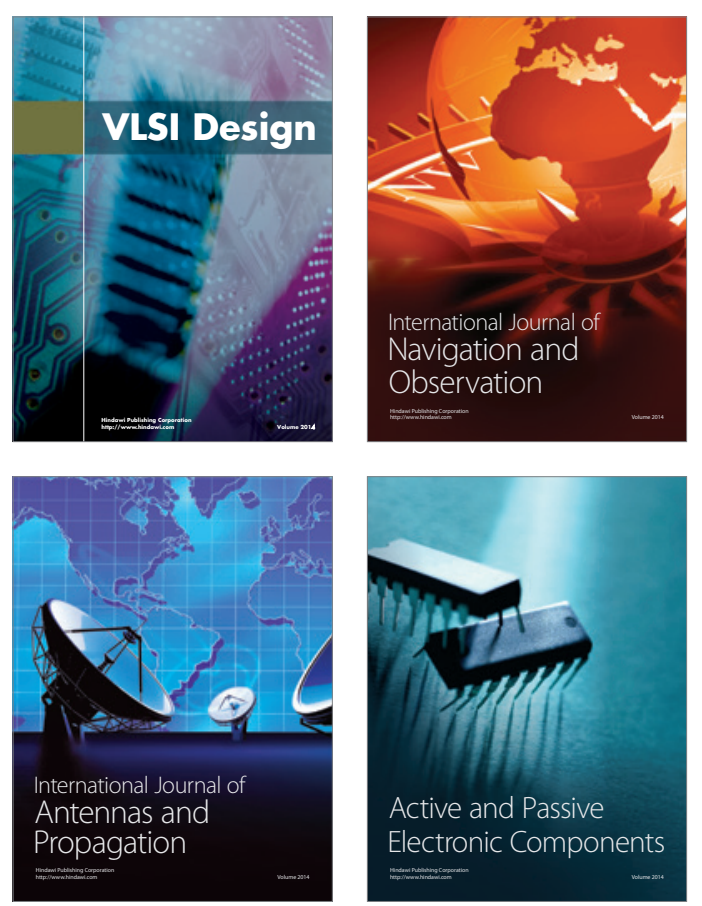
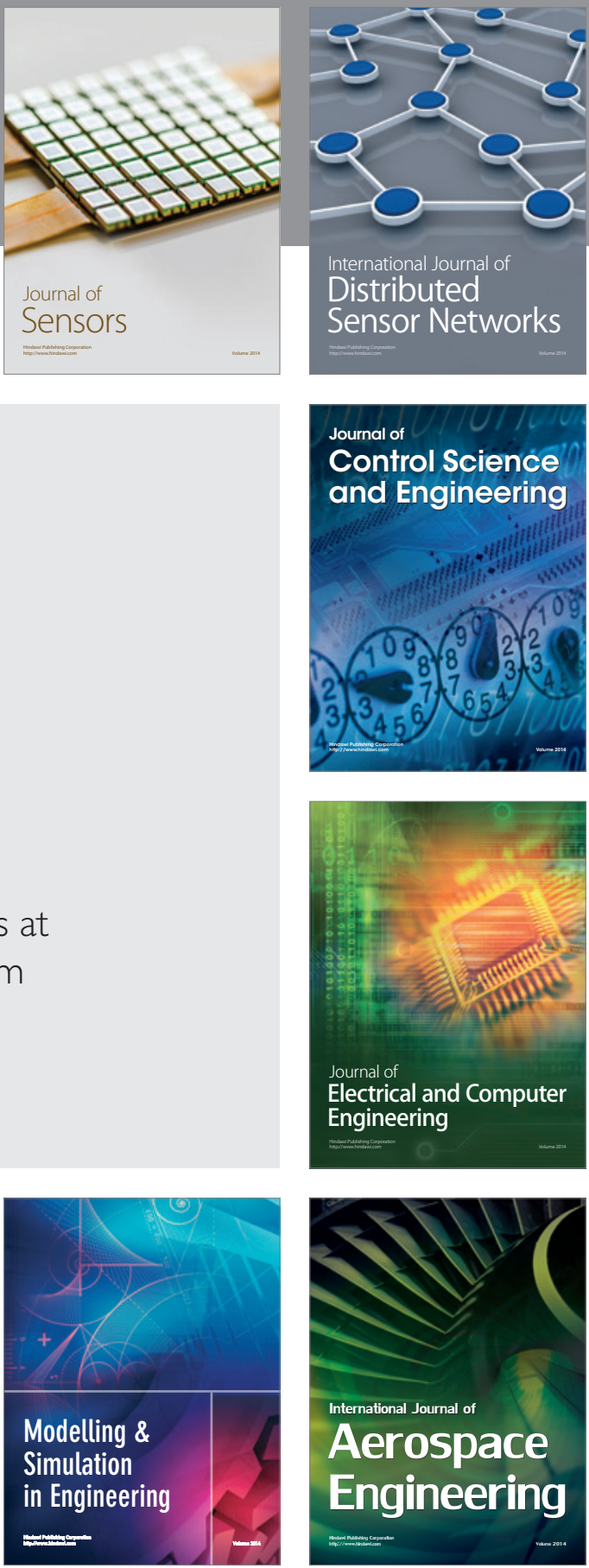

Journal of

Control Science

and Engineering
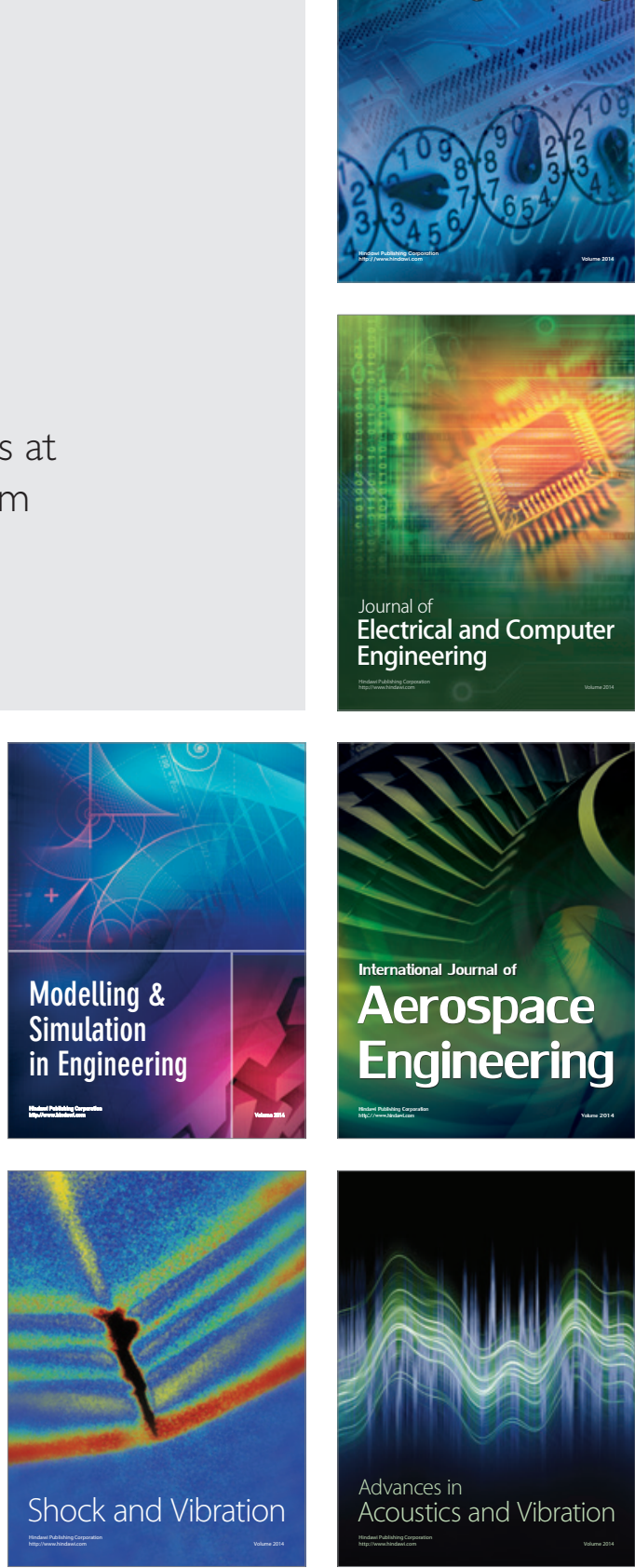